\title{
DETERMINANTAL HYPERSURFACES AND REPRESENTATIONS OF COXETER GROUPS
}

\author{
Ž. ČUČKOVIČ, M. I. STESSIN, AND A. B. TCHERNEV
}

\begin{abstract}
Given a finite generating set $T=\left\{g_{0}, \ldots, g_{n}\right\}$ of a group $G$, and a representation $\rho$ of $G$ on a Hilbert space $V$, we investigate how the geometry of the set $D(T, \rho)=\left\{\left[x_{0}: \cdots: x_{n}\right] \in \mathbb{C P}^{n} \mid \sum x_{i} \rho\left(g_{i}\right)\right.$ not invertible $\}$ reflects the properties of $\rho$. When $V$ is finite-dimensional this is an algebraic hypersurface in $\mathbb{C P}^{n}$. In the special case $T=G$ and $\rho=$ the left regular representation of $G$, this hypersurface is defined by the group determinant, an object studied extensively in the founding work of Frobenius that lead to the creation of representation theory. We focus on the classic case when $G$ is a finite Coxeter group, and make $T$ by adding the identity element $1_{G}$ to a Coxeter generating set for $G$. Under these assumptions we show in our first main result that if $\rho$ is the left regular representation, then $D(T, \rho)$ determines the isomorphism class of $G$. Our second main result is that if $G$ is not of exceptional type, and $\rho$ is any finite dimensional representation, then $D(T, \rho)$ determines $\rho$.
\end{abstract}

\section{INTRODUCTION}

Given a tuple of linear operators $A_{0}, \ldots, A_{n}$, on a complex $N$-dimensional vector space $V$, the determinant

$$
\mathcal{P}\left(x_{0}, \ldots, x_{n}\right)=\operatorname{det}\left[x_{0} A_{0}+\cdots+x_{n} A_{n}\right]
$$

is a homogeneous polynomial in $x_{0}, \ldots, x_{n}$ of degree $N$. Zeros of this polynomial form an algebraic closed subscheme of complex projective space $\mathbb{C P}^{n}$ called a determinantal hypersurface, and we denote it by $\sigma\left(A_{0}, \ldots, A_{n}\right)$.

Determinantal hypersurfaces are objects with a long history in algebraic geometry. A major direction of research related to their study is to determine which hypersurfaces in $\mathbb{C P}^{n}$ are determinantal. This is a classical avenue, see e.g. [4, 12, 13, 23, 24, 30, 34, 39.

In this paper we follow another approach to determinantal hypersurfaces, where one analyzes what the geometry of $\sigma\left(A_{0}, \ldots, A_{n}\right)$ can say about the mutual relations between the operators $A_{0}, \ldots, A_{n}$. While this kind of question is natural from the perspective of operator theory, until recently it seems to have attracted much less attention in geometry.

One of the few relevant instances of work along these lines is the result of Motzkin and Taussky 32 that a real curve in $\mathbb{C P}^{2}$ having a self-adjoint determinantal representation with one of the three operators being invertible (and, therefore, assumed to be the identity) satisfies the condition: the operators commute if and only if

2010 Mathematics Subject Classification. Primary: 20F55, 20C33, 14J70, 47A25; Secondary: $47 \mathrm{~A} 67$.

Key words and phrases. determinantal hypersurfaces, Coxeter groups, joint spectrum, group representations. 
this determinantal curve is a union of projective lines (in 32 the result is stated in equivalent but different form).

Another - and the most relevant to the subject of this paper - instance where the geometry of a determinantal hypersurface has been extensively studied from our point of view comes from classic work of Frobenius that goes back to the origins of representation theory. Specifically, when $G=\left\{g_{0}, \ldots, g_{n}\right\}$ is a finite group and $A_{i}$ represents the action of $g_{i}$ by left multiplication on the group ring $\mathbb{C}[G]$ (this is usually called the left regular representation of $G$ ), the defining equation of $\sigma\left(A_{0}, \ldots, A_{n}\right)$ is called the group determinant of $G$. The papers of Frobenius [17, 18, 19] show, in modern language, that the irreducible components of $\sigma\left(A_{0}, \ldots, A_{n}\right)$ are in bijective correspondence with the irreducible representations of $G$, and the multiplicity of each component equals the dimension of the corresponding irreducible representation. For more on the group determinant and related problems we refer the reader to [8, 9, 10, 11, 16, 26, 27, 29] and the references there. In particular, it was shown by Formanek and Sibley [16] that the group determinant determines the isomorphism class of the group as well.

A substantial drawback when working with group determinants is that, as the size of the group rises, the resulting increase in complexity makes the group determinant essentially inaccessible. It is natural to ask if it is possible, instead of all elements, to take only a suitable generating set for $G$, thus computing a much simpler determinantal hypersurface while still retaining all the salient features of the group determinant. Furthermore, it is desirable to have analogous results also for finitely generated groups that are not necessarily finite. To do this one would need a generalization for the notion of a determinantal hypersurface to the setting of operators on an infinite dimensional space. This naturally leads us to the notion of projective joint spectrum for operators acting on a Hilbert space $V$, which was introduced by Yang [40. If $A_{0}, \ldots, A_{n}$ are bounded linear operators on $V$, their joint spectrum is the set

$$
\sigma\left(A_{0}, \ldots, A_{n}\right)=\left\{\left[x_{0}: \cdots: x_{n}\right] \in \mathbb{C P}^{n}: x_{0} A_{0}+\cdots+x_{n} A_{n} \text { is not invertible }\right\} .
$$

When $V$ is finite dimensional, the joint spectrum is a determinantal hypersurface. Since in this paper we predominantly deal with the finite dimensional case, we will use the terms "determinantal hypersurface" and "joint spectrum" interchangeably. The relation between the geometry of the joint spectrum (or its complement, the joint resolvent set) and the properties of the tuple $A_{0}, \ldots, A_{n}$ was investigated in $2,3,5,14,15,21,22,25,37,36,40$. In 22 joint spectrum was used in relation to the Gelfand-Naimark-Siegal representation of the infinite dihedral group.

Let $T=\left\{g_{0}, \ldots, g_{n}\right\}$ be a set of generators of a group $G$, and let $\rho: G \longrightarrow G L(V)$ be a homomorphism into the group of bounded invertible linear operators on a Hilbert space $V$. Let

$$
D(T, \rho)=\sigma\left(\rho\left(g_{0}\right), \ldots, \rho\left(g_{n}\right)\right)
$$

be the corresponding joint spectrum. If $\rho$ is the left regular representation of $G$ we will write just $D(T)$ (when $G$ is infinite its left regular representation is obtained by the left action of $G$ on the completion of $\mathbb{C}[G]$ with respect to its inner product norm, see Section 2). The results of Frobenius and of Formanek and Sibley naturally prompt us to ask:

(1) When does $D(T)$ determine $G$ ?

(2) When does $D(T, \rho)$ determine $\rho$ ? 
(3) If $\rho$ is finite dimensional and irreducible, when is $D(T, \rho)$ reduced and irreducible?

We address these fundamental structural questions in the classical case when $G$ is a finitely generated Coxeter group. We consider the generating set $T$ obtained by adding the identity element $1_{G}$ to a Coxeter generating set for $G$; see Section 2 for definitions. Our first main result concerns question (1):

Theorem 1.1. Let $G$ be a Coxeter group with Coxeter generating set $\left\{g_{1}, \ldots, g_{n}\right\}$, and let $T=\left\{1_{G}, g_{1}, \ldots, g_{n}\right\}$. Let $G^{\prime}$ be a group, and let $T^{\prime}=\left\{1_{G^{\prime}}, g_{1}^{\prime}, \ldots, g_{n}^{\prime}\right\}$ be a generating set for $G^{\prime}$. We have:

(1) If $D(T) \supseteq D\left(T^{\prime}\right)$ as subsets of $\mathbb{C P}^{n}$, then there is an epimorphism of groups $f: G \longrightarrow G^{\prime}$ such that $f\left(g_{i}\right)=g_{i}^{\prime}$ for each $1 \leq i \leq n$. In particular, if $G$ is finite then so is $G^{\prime}$.

(2) If $G$ is finite and $D(T)=D\left(T^{\prime}\right)$ as subschemes of $\mathbb{C P}^{n}$, then the homomorphism $f$ from (1) is an isomorphism.

Next, we consider question (2) for finite dimensional representations. It is easy to see that, when $V$ is finite dimensional, $D(T, \rho)$ is completely determined by the multiset of isomorphism classes of irreducible representations that appear as factors in a composition series for $V$ when considered as a $\mathbb{C}[G]$-module via $\rho$. Thus whenever $\mathbb{C}[G]$ is not a semisimple ring, there will always exist non-equivalent representations of $G$ having the same determinantal hypersurface. Note however, that this kind of obstruction does not arise if $G$ is finite, or if one considers either only unitary or only irreducible representations.

In our second main result we present a positive answer to question (2) when $G$ is a finite Coxeter group of regular type. The exceptional types will be treated in a separate paper.

Theorem 1.2. Let $G$ and $T$ be as in Theorem 1.1. Suppose $G$ is finite of type either I (dihedral group), or $A$, or $B$, or $D$. If for two finite dimensional complex linear representations $\rho_{1}$ and $\rho_{2}$ of $G$ we have

$$
D\left(T, \rho_{1}\right)=D\left(T, \rho_{2}\right)
$$

as subschemes of $\mathbb{C P}^{n}$, then the representations $\rho_{1}$ and $\rho_{2}$ are equivalent.

In our proofs we make a conscious attempt to emphasize the direct connection between porperties of the generating set $T$ and the geometry of the corresponding determinantal hypersurface. Thus, in the case when $G$ is dihedral, even though there are alternative arguments using known results from representation theory, we chose to rely on the tools developed in the recent paper 37, where the relation between the appearance of algebraic curves of finite multiplicity in the joint spectrum of a tuple of operators and decomposability of the tuple is investigated. In the case of unitary self-adjoint operators the technique developed there can be applied to finding commutation relations between the operators, and, therefore, be applied to representations of Coxeter groups. This method is quite general, and proved to be useful also for other, non-Coxeter groups. The upcoming paper 38 will contain the details.

To handle the cases $A, B$, and $D$ in Theorem 1.2, we demonstrate that in those types $D(T, \rho)$ encodes the character of the representation $\rho$. We accomplish this by leveraging an explicit combinatorial algorithm that works uniformly in all three regular types, and transforms an element $g$ of $G$ into what we call echelon form - 
an element with good combinatorial properties that belongs to the same conjugacy class, and is, in a precise sense, no bigger than $g$. The resulting combinatorics seems to be new and may be of independent interest.

The structure of the paper is as follows. Section 2 provides definitions and basic results about group representations and Coxeter groups. Section 3 contains the needed background material and results regarding joint spectra.

In Section 4 we analyze joint spectra of involution pairs. The computations reveal an interesting connection to Tchebyshev's polynomials. We use these results and give the proof of Theorem 1.1.

In Section 5 we work with finite dimensional unitary representations of dihedral groups. We demonstrate how one can use the tools from 37] to construct explicitly the decomposition of such a representation into irreducible factors directly from the defining equation of the joint spectrum. We use that to give a proof of Theorem 1.2 for the case when $G$ is finite dihedral, and prove an analogous result for unitary representations of the infinite dihedral group. We also address question (3) for that setting. Of course, since the irreducible representations of dihedral groups are well known, these results can also be obtained by direct analysis of the determinantal hypersurfaces of these irreducible representations.

Sections [6] and 7 are devoted to developing the combinatorics needed for the proof of Theorem 1.2 for $G$ of types $A, B$, or $D$. Section [6] considers words in the alphabet of the Coxeter generators of $G$, and introduces a partial ordering and admissible transformations on these words. We show that admissible transformations preserve conjugacy classes, and are non-increasing with respect to the partial ordering. Section 7 introduces echelon forms. The main result there is the "Ordering Theorem", Theorem 7.5, which states that every word can be transformed into echelon form via a sequence of admissible transformations. This result is the key to our proof of Theorem 1.2, which we present in Section 8.

The combinatorics of admissible transformations can be extended to other Coxeter groups. In Section 9 we give an example how this can be used to study representations of the affine Coxeter group $\tilde{C}_{2}$ via determinantal hypersurfaces.

\section{Preliminaries}

Let $G$ be group. Recall that a (complex) representation of $G$ is a group homomorphism $\rho: G \longrightarrow G L(V)$ from $G$ to the group of bounded invertible linear operators on a Hilbert space $V$. The representation $\rho$ is called unitary if $\rho(w)$ is unitary for all $w \in G$; it is called finite dimensional if $V$ is finite dimensional; and it is called faithful if $\rho$ is injective. Two representations $\rho_{1}: G \rightarrow G L\left(V_{1}\right)$ and $\rho_{2}: G \rightarrow G L\left(V_{2}\right)$ are equivalent if there is a bounded linear isomorphism $C: V_{1} \rightarrow V_{2}$ such that $\rho_{2}(w)=C \rho_{1}(w) C^{-1}$ for every $w \in G$.

The group ring $\mathbb{C}[G]$ has inner product $\left\langle\sum_{g \in G} a_{g} g, \sum_{g \in G} b_{g} g\right\rangle=\sum_{g \in G} a_{g} \bar{b}_{g}$ and corresponding induced norm $\left\|\sum a_{g} g\right\|=\sum\left|a_{g}\right|^{2}$. We write $\mathbb{C}[G]^{\vee}$ for the Hilbert space obtained by completing $\mathbb{C}[G]$ with respect to this norm. Of course, if $G$ is finite then $\mathbb{C}[G]^{\vee}=\mathbb{C}[G]$. For each $g \in G$ the left multiplication by $g$ on $\mathbb{C}[G]$ induces a bounded invertible linear operator $\lambda(g)$ on $\mathbb{C}[G]^{\vee}$, and the resulting map $\lambda: G \rightarrow G L\left(\mathbb{C}[G]^{\vee}\right)$ is a faithful unitary representation of $G$ called the left regular representation.

When dealing with representations of finite groups we will rely heavily on the following basic fact (see 35]). 
Theorem 2.1. Every linear representation of a finite group $G$ is equivalent to a unitary representation.

An important invariant of a finite dimensional representation $\rho$ of any group $G$ is the character $\chi_{\rho}$ of $\rho$. This is a function on $G$ defined by

$$
\chi_{\rho}(w)=\operatorname{Tr}(\rho(w)) .
$$

The character of $\rho$ is a class-function, that is, it is constant on conjugacy classes. In fact, when $G$ is finite it determines completely $\rho$ (see [35]):

Theorem 2.2. Let $G$ be a finite group, and $\rho_{1}$ and $\rho_{2}$ be two finite dimensional representations of $G$. If $\chi_{\rho_{1}}=\chi_{\rho_{2}}$, then $\rho_{1}$ and $\rho_{2}$ are equivalent.

Next, we review some basics on Coxeter groups. The monographs [1], 20], and 28] are good sources for their combinatorics, and properties.

A Coxeter group is a finitely generated group $G$ on generators $g_{1}, \ldots, g_{n}$ defined by the following relations:

$$
\left(g_{i} g_{j}\right)^{m_{i j}}=1, \quad i, j=1, \ldots, n,
$$

where $m_{i i}=1$ and $m_{i j} \in \mathbb{N} \cup\{\infty\}$, with $m_{i j} \geq 2$ when $i \neq j$. It is easy to see that to avoid redundancy we must have $m_{i j}=m_{j i}$, and that $m_{i j}=2$ means $g_{i}$ and $g_{j}$ commute. The set of generators $\left\{g_{1}, \ldots, g_{n}\right\}$ is called a Coxeter set of generators, and the $m_{i j}$ s are called the Coxeter exponents.

A traditional way of presentation of a Coxeter group is through its Coxeter diagram, which is a graph constructed by the following rules:

- the vertices of the graph are the generator subscripts;

- vertices $i$ and $j$ form an edge if and only if $m_{i j} \geq 3$;

- an edge is labeled with the value $m_{i j}$ whenever this value is 4 or greater.

In particular, two generators commute if and only if they are not connected by an edge. The disjoint union of Coxeter diagrams yields a direct product of Coxeter groups, and a Coxeter group is connected if its diagram is a connected graph.

The finite connected Coxeter groups consist of the one-parameter families $A_{n}$, $B_{n}, D_{n}$, and $I(n)$, and the six exceptional groups $E_{6}, E_{7}, E_{8}, F_{4}, H_{3}$, and $H_{4}$. They were classified by Coxeter [7, and in [6] Coxeter proved that every reflection group is a Coxeter group.

The Coxeter diagrams for the groups $A_{n}, B_{n}, D_{n+1}$, and $I(n)$ that we study in this paper are as follows:

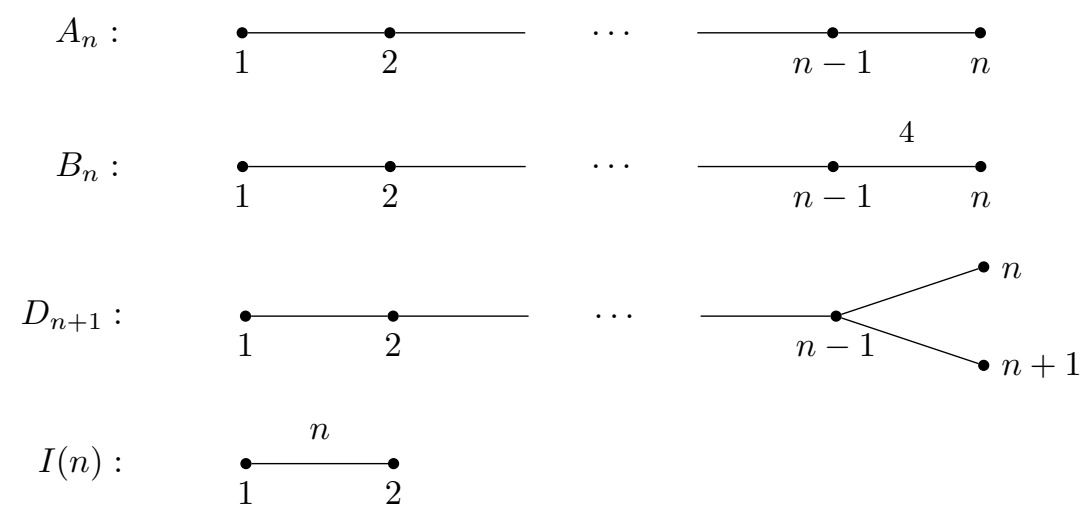




\section{MORE ON JOINT SPECTRA}

Recall that for an algebraic hypersurface in $\mathbb{C P}^{n}$ or in $\mathbb{C}^{n}$ defined by a polynomial $F=F_{1}^{r_{1}} \ldots F_{m}^{r_{m}}$ (with each polynomial $F_{i}$ irreducible and $F_{i}$ not associate with $F_{j}$ for $i \neq j$ ), the components of that hypersurface are defined by the polynomials $F_{i}^{r_{i}}$ (thus they are irreducible but not necessarily reduced), the reduced components are defined by the polynomials $F_{i}$, and the exponent $r_{i}$ is called the multiplicity of the reduced component defined by $F_{i}$. We say that a point on our hypersurface is regular (with multiplicity $r$ ) if it belongs to only one reduced component (of multiplicity $r$ ), and is a regular point on that reduced component.

Let $A$ be a bounded linear operator on a Hilbert space $V$. We consider its norm given by $\|A\|=\sup _{|u|=1}|A(u)|$. The spectrum of $A$ is the set

$$
\sigma(A)=\{\lambda \in \mathbb{C} \mid A-\lambda I \text { is not invertible }\} .
$$

Note that when $A$ is self-adjoint we have $\sigma(A) \subseteq \mathbb{R}$ and $\|A\|=\sup _{\lambda \in \sigma(A)}|\lambda|$. We will use the following elementary consequences of the Spectral Theorem and the Spectral Mapping Theorem, see 31.

Proposition 3.1. Let $A$ be a bounded linear operator on a Hilbert space $V$.

(a) If $p(x)$ is any polynomial then $\sigma(p(A))=p(\sigma(A))=\{p(\lambda) \mid \lambda \in \sigma(A)\}$.

(b) If $A$ is normal and $\sigma(A)=\{1\}$ then $A=I$, the identity operator.

Now let $A_{1}, A_{2}, \ldots, A_{n}$ be bounded linear operators on a Hilbert space $V$. We will be considering the projective joint spectrum $\sigma\left(-I, A_{1}, \ldots, A_{n}\right)$ of the tuple $-I, A_{1}, A_{2}, \ldots, A_{n}$, where $I$ is the identity operator, and its intersection with the chart $\left\{\left[x_{0}: \cdots: x_{n}\right] \mid x_{0} \neq 0\right\}$. By taking $x_{0}=1$ we identify this intersection with a closed subset of $\mathbb{C}^{n}$ called the proper joint spectrum of the tuple $A_{1}, \ldots, A_{n}$, denoted by $\sigma_{p}\left(A_{1}, \ldots, A_{n}\right)$; and when $V$ is finite dimensional its defining polynomial is

$$
\mathcal{F}\left(x_{1}, \ldots, x_{n}\right)=\operatorname{det}\left(-I+x_{1} A_{1}+\cdots+x_{n} A_{n}\right) .
$$

In particular, a point $x=\left(x_{1}, \ldots, x_{n}\right) \in \mathbb{C}^{n}$ belongs to the proper joint spectrum if and only if the operator $A(x)=x_{1} A_{1}+\cdots+x_{n} A_{n}$ has 1 in its spectrum. We note that when $V$ is finite dimensional and $\sigma_{p}\left(A_{1}, \ldots, A_{n}\right)$ is not empty (this latter is the case, for example, if $A_{i}$ is invertible for some $i$ ), the projective joint spectrum $\sigma\left(-I, A_{1}, \ldots, A_{n}\right)$ is the closure of the determinantal hypersurface $\sigma_{p}\left(A_{1}, \ldots, A_{n}\right)$, and its defining polynomial is the homogenization of $\mathcal{F}\left(x_{1}, \ldots, x_{n}\right)$.

We will be using the following basic observation multiple times:

Proposition 3.2. Suppose $V$ is finite dimensional, let $u=\left(u_{1}, u_{2}\right)$ be a regular point of $\sigma_{p}\left(A_{1}, A_{2}\right)$ of multiplicity $r$, and let $\Gamma$ be the corresponding unique reduced component containing $u$.

If the tangent line to $\Gamma$ at $u$ does not pass through the origin $(0,0)$ then the operator $A(u)=u_{1} A_{1}+u_{2} A_{2}$ has eigenvalue 1 of multiplicity exactly $r$.

Proof. Indeed, let $\mathcal{F}\left(x_{1}, x_{2}\right)=\operatorname{det}\left(-I+x_{1} A_{1}+x_{2} A_{2}\right)=F^{r} H$ be the defining polynomial of $\sigma_{p}\left(A_{1}, A_{2}\right) \neq \emptyset$, where $F$ is a defining irreducible polynomial of $\Gamma$, and $H$ is not divisible by $F$. Then the defining polynomial $\mathcal{P}\left(x_{0}, x_{1}, x_{2}\right)=$ $\operatorname{det}\left(-x_{0} I+x_{1} A_{1}+x_{2} A_{2}\right)=\tilde{F}^{r} \tilde{H}$ of $\sigma\left(-I, A_{1}, A_{2}\right)$ is the homogenization of $\mathcal{F}$, where $\tilde{F}$ and $\tilde{H}$ are the homogenizations of $F$ and $H$. Note $\mathcal{P}$ is also (as a polynomial in $x_{0}$ ) the characteristic polynomial of the operator $A(x)$. Thus we have $\mathcal{P}\left(x_{0}, u_{1}, u_{2}\right)=\tilde{F}^{r}\left(x_{0}, u_{1}, u_{2}\right) \tilde{H}\left(x_{0}, u_{1}, u_{2}\right)=\left(1-x_{0}\right)^{m} \prod_{i=1}^{k}\left(\lambda_{i}-x_{0}\right)^{m_{i}}$ where the 
$\lambda_{i}$ are the eigenvalues of $A(u)$ different from 1 , and the $m_{i}$ their multiplicities. Since $u$ is only on $\Gamma$, it follows that $\tilde{H}\left(1, u_{1}, u_{2}\right)=H(u) \neq 0$, hence $\left(1-x_{0}\right)^{m}$ is a factor of $\tilde{F}^{r}\left(x_{0}, u_{1}, u_{2}\right)$. Thus it suffices to check that $x_{0}=1$ is not a double root of $\tilde{F}\left(x_{0}, u_{1}, u_{2}\right)$ when considered as a polynomial in $x_{0}$. Since for the corresponding partial derivative in $x_{0}$ we have $\tilde{F}_{x_{0}}\left(1, u_{1}, u_{2}\right)=-u_{1} F_{x_{1}}\left(u_{1}, u_{2}\right)-u_{2} F_{x_{2}}\left(u_{1}, u_{2}\right)$, and the equation for the tangent line to $\Gamma$ at $u$ is $\left(x_{1}-u_{1}\right) F_{x_{1}}(u)+\left(x_{2}-u_{2}\right) F_{x_{2}}(u)=0$, the desired conclusion is immedaite from our assumption that the tangent line does not contain the origin.

It was shown in 37] that substantial information regarding mutual relations between the $A_{i}$ s is captured by the geometry of their proper joint spectrum, and we review some of these results below, in the case of two operators $A_{1}$ and $A_{2}$.

First we note the behaviour of the joint spectrum under change of coordinates. Let

$$
\mathbf{C}=\left[\begin{array}{ll}
c_{11} & c_{12} \\
c_{21} & c_{22}
\end{array}\right]
$$

be an invertible complex-valued matrix. Write

$$
B_{1}=c_{11} A_{1}+c_{12} A_{2}, \quad B_{2}=c_{21} A_{1}+c_{22} A_{2} .
$$

Then it is immediate from the definitions that

$$
\sigma_{p}\left(A_{1}, A_{2}\right)=\mathbf{C}^{T} \sigma_{p}\left(B_{1}, B_{2}\right) .
$$

Next, suppose $V$ is finite dimensional and $A_{1}$ is self-adjoint. Let $\lambda \neq 0$ be an eigenvalue of $A_{1}$, and suppose that $(1 / \lambda, 0)$ is a regular point of the determinantal hypersurface $\sigma_{p}\left(A_{1}, A_{2}\right)$. Let $\mathcal{R}\left(x_{1}, x_{2}\right)$ be a defining polynomial for the reduced component of $\sigma_{p}\left(A_{1}, A_{2}\right)$ that contains $(1 / \lambda, 0)$; thus one of the partial derivatives, say, $\mathcal{R}_{x_{1}}=\frac{\partial \mathcal{R}}{\partial x_{1}}$, does not vanish at $(1 / \lambda, 0)$. Then the implicit function theorem implies that in a neighborhood of $(1 / \lambda, 0)$ the equation $\{\mathcal{R}=0\}$ determines $x_{1}$ as an implicit analytic function of $x_{2}$ defined in a neighborhood of the origin:

$$
x_{1}=x_{1}\left(x_{2}\right), \quad x_{1}(0)=\frac{1}{\lambda} .
$$

For each eigenvalue $w$ in the spectrum $\sigma\left(A_{1}\right)$ of $A_{1}$ let $P_{w}$ be the orthogonal projection onto the $w$-eigensubspace of $A_{1}$. We also set $P=P_{\lambda}$. Since $A_{1}$ is self-adjoint, the spectral decomposition for $A_{1}$ is

$$
A_{1}=\lambda P+\sum_{w \in \sigma\left(A_{1}\right) \backslash\{\lambda\}} w P_{w} .
$$

We also introduce the following operator:

$$
T\left(A_{1}\right)=T=\sum_{w \in \sigma\left(A_{1}\right) \backslash\{\lambda\}} \frac{\lambda}{w-\lambda} P_{w} .
$$

The following result was proved (in significantly greater generality) during the course of [37, Proof of Theorem 7.3].

Theorem 3.8. Suppose that $V$ is finite dimensional, that $A_{1}$ and $A_{2}$ are selfadjoint, and that $\lambda \neq 0$ is an eigenvalue of $A_{1}$ such that

- $(1 / \lambda, 0)$ is a regular point of $\sigma_{p}\left(A_{1}, A_{2}\right)$; and

- $\mathcal{R}_{x_{1}}(1 / \lambda, 0) \neq 0$, where $\mathcal{R}\left(x_{1}, x_{2}\right)$ is a defining polynomial for the reduced component of $\sigma_{p}\left(A_{1}, A_{2}\right)$ containing $(1 / \lambda, 0)$. 
Then we have

$$
P A_{2} P=-x_{1}^{\prime}(0) P
$$

and

$$
P A_{2} T A_{2} P=-\frac{x_{1}^{\prime \prime}(0)}{2} P,
$$

where we regard $x_{1}$ as an implicit analytic function (3.5) of $x_{2}$ defined via the equation $\mathcal{R}=0$.

\section{Joint SPECTRA AND COXETER GROups}

Here we study properties of joint spectra of involution pairs $A_{1}, A_{2}$, and use these to prove Theorem 1.1 .

Lemma 4.1. Let $A_{1}$ and $A_{2}$ be bounded linear operators on a Hilbert space $V$ such that $A_{1}^{2}=A_{2}^{2}=I$. Then:

(1) The set $\sigma_{p}\left(A_{1}, A_{2}\right) \cup-\sigma_{p}\left(A_{1}, A_{2}\right)$ is the union of all the "complex ellipses" $\mathcal{E}_{\alpha}=\left\{x^{2}+\alpha x y+y^{2}=1\right\}$ with $\alpha \in \sigma\left(A_{1} A_{2}+A_{2} A_{1}\right)$.

(2) When $\sigma\left(A_{1} A_{2}+A_{2} A_{1}\right)$ is a finite set then each connected component of $\sigma_{p}\left(A_{1}, A_{2}\right) \backslash\{( \pm 1,0)(0, \pm 1)\}$ is either $L \backslash\{( \pm 1,0)(0, \pm 1)\}$ with $L$ one of the lines $x \pm y= \pm 1$, or $\mathcal{E}_{\alpha} \backslash\{( \pm 1,0)(0, \pm 1)\}$ for some $\alpha \in \sigma\left(A_{1} A_{2}+A_{2} A_{1}\right)$.

(3) When $V$ is finite dimensional each reduced component of $\sigma_{p}\left(A_{1}, A_{2}\right)$ is either a line of the form $x \pm y= \pm 1$, or a "complex ellipse" $\mathcal{E}_{\alpha}$ with $\alpha \in \sigma\left(A_{1} A_{2}+A_{2} A_{1}\right) \backslash\{-2,2\}$.

Proof. Suppose that $(x, y) \in \sigma_{p}\left(A_{1}, A_{2}\right) \cup-\sigma_{p}\left(A_{1}, A_{2}\right)$. This is if and only if $1 \in \sigma\left(x A_{1}+y A_{2}\right)$ or $-1 \in \sigma_{p}\left(x A_{1}+y A_{2}\right)$, which, by Proposition 3.1, happens precisely when $1 \in \sigma\left(\left(x A_{1}+y A_{2}\right)^{2}\right)$. That in turn happens if and only if the operator

$$
\left(x A_{1}+y A_{2}\right)^{2}-I=x y\left[A_{1} A_{2}+A_{2} A_{1}\right]-\left(1-x^{2}-y^{2}\right) I
$$

is not invertible. We see that when $x y \neq 0$ this is equivalent to $\alpha=\frac{1-x^{2}-y^{2}}{x y}$ being in the spectrum of $A_{1} A_{2}+A_{2} A_{1}$, and $(x, y)$ being a point on $\mathcal{E}_{\alpha}$ (and this last fact is true for trivial reasons also when $x y=0$ ). This completes the proof of part (1).

When $\sigma\left(A_{1} A_{2}+A_{2} A_{1}\right)$ is a finite set equation (4.2) implies that $\frac{1-x^{2}-y^{2}}{x y}$ is constant on every connected component of $\sigma_{p}\left(A_{1}, A_{2}\right) \backslash\{( \pm 1,0)(0, \pm 1)\}$, which yields (2). Therefore, if $V$ is finite dimensional, then each reduced component of $\sigma_{p}\left(A_{1}, A_{2}\right)$ is a reduced component of an ellipse $\mathcal{E}_{\alpha}$ for some $\alpha \in \sigma\left(A_{1} A_{2}+A_{2} A_{1}\right)$. When $\alpha \neq \pm 2$ the ellipse is irreducible, so our reduced component coincides with the ellipse. When $\alpha= \pm 2$ the ellipse is the union of two of the lines $x \pm y= \pm 1$, hence our reduced component is one of these lines.

Lemma 4.3. Let $A_{1}$ and $A_{2}$ be as in Lemma 4.1, and let $m \geq 2$ be an integer. If

(1) $\left(A_{1} A_{2}\right)^{m}=I$ then

(2) $\sigma\left(A_{1} A_{2}+A_{2} A_{1}\right) \subseteq\{2 \cos (2 \pi k / m) \mid k=0, \ldots, m-1\}$.

If both $A_{1}$ and $A_{2}$ are unitary, then conditions (1) and (2) are equivalent. 
Proof. For each $n \geq 0$ set $R_{n}=(1 / 2)\left[\left(A_{1} A_{2}\right)^{n}+\left(A_{2} A_{1}\right)^{n}\right]$. It is straightforward to check that

$$
\begin{aligned}
& R_{0}=I, \\
& R_{1}=(1 / 2)\left(A_{1} A_{2}+A_{2} A_{1}\right), \quad \text { and } \\
& R_{n}=2 R_{1} R_{n-1}-R_{n-2} \quad \text { for } n \geq 2 .
\end{aligned}
$$

It follows immediately by induction that for each $n \geq 0$ we have

$$
R_{n}=T_{n}\left(R_{1}\right),
$$

where $T_{n}(z)$ are Tchebyshev's polynomials of the first kind defined by

$$
\begin{aligned}
& T_{0}(z)=1, \\
& T_{1}(z)=z, \quad \text { and } \\
& T_{n}(z)=2 z T_{n-1}(z)-T_{n-2}(z) \quad \text { for } n \geq 2 .
\end{aligned}
$$

It is well known that for each real $z \in[-1,1]$ one has $T_{n}(z)=\cos \left(n \cos ^{-1}(z)\right)$, cf. Rivlin [33, in particular the polynomial $T_{n}(z)-1$ is of degree $n$ and has for its set of roots the set $\{\cos (2 \pi k / n) \mid k=0, \ldots n-1\}$.

Now, suppose $\left(A_{1} A_{2}\right)^{m}=I$. Thus $\left(A_{2} A_{1}\right)^{m}=I$ as well, hence $R_{m}=T_{m}\left(R_{1}\right)=$ I. Since by Proposition 3.1(a) we have $\sigma\left(R_{m}\right)=T_{m}\left(\sigma\left(R_{1}\right)\right)$, we must have $T_{m}(\alpha)=$ 1 for each $\alpha \in \sigma\left(R_{1}\right)$. Therefore $\sigma\left(R_{1}\right) \subseteq\{\cos (2 \pi k / m) \mid k=0, \ldots, m-1\}$, which implies (2) as desired.

Next, suppose $A_{1}$ and $A_{2}$ are both unitary and (2) holds. Then $R_{n}$ is self-adjoint for each $n$. In particular $R_{m}$ is self-adjoint and has $\sigma\left(R_{m}\right)=T_{m}\left(\sigma\left(R_{1}\right)\right)=\{1\}$. It follows from Proposition 3.1(b) that $R_{m}=I$. Thus $\left(A_{1} A_{2}\right)^{m}+\left(A_{1} A_{2}\right)^{-m}=2 I$ hence $\left(A_{1} A_{2}\right)^{2 m}-2\left(A_{1} A_{2}\right)^{m}+I=0$. Proposition 3.1 (a) now yields that $(\lambda-1)^{2}=0$ for every $\lambda \in \sigma\left(\left(A_{1} A_{2}\right)^{m}\right)$. So $\left(A_{1} A_{2}\right)^{m}$ is a unitary hence normal operator whose spectrum is the singleton $\{1\}$, and therefore (1) holds by Proposition 3.1(b).

Remark 4.4. The above proofs show that the spectral decomposition of the operator $A_{1} A_{2}+A_{2} A_{1}$ determines the decomposition of the pair $\left(A_{1}, A_{2}\right)$ and the decomposition of the joint spectrum of $A_{1}$ and $A_{2}$ into irreducible components. The fact that $A_{1} A_{2}+A_{2} A_{1}$ commutes with both $A_{1}$ and $A_{2}$, and, therefore, belongs to the center of the group-algebra, plays a key role here.

Now we are ready to present the proof of Theorem 1.1

Proof of Theorem 1.1. We have $T=\left\{1_{G}, g_{1}, \ldots, g_{n}\right\}$ where $\left\{g_{1}, \ldots, g_{n}\right\}$ is a system of Coxeter generators of for $G$. Also, we have $T^{\prime}=\left\{1_{G^{\prime}}, g_{1}^{\prime}, \ldots, g_{n}^{\prime}\right\}$ where $\left\{g_{1}^{\prime}, \ldots, g_{n}^{\prime}\right\}$ is a generating set for the group $G^{\prime}$. Let $A_{i}$ (resp. $A_{i}^{\prime}$ ) be the operator on $\mathbb{C}[G]^{\vee}$ (resp. $\mathbb{C}\left[G^{\prime}\right]^{\vee}$ ) induced via left multiplictaion by $g_{i}$ (resp. $g_{i}^{\prime}$ ) on the group ring $\mathbb{C}[G]$ (resp. $\mathbb{C}\left[G^{\prime}\right]$ ). Suppose $D(T) \supseteq D\left(T^{\prime}\right)$. We also have $\sigma_{p}\left(A_{1}, \ldots, A_{n}\right) \supseteq \sigma_{p}\left(A_{1}^{\prime}, \ldots, A_{n}^{\prime}\right)$. Furthermore, for all $i$ and all $k<l$ intersecting with the projective line $\left\{x_{j}=0 \mid 0 \neq j \neq i\right\}$, and with the projective plane $\left\{x_{j}=0 \mid j \notin\{0, k, l\}\right\}$ shows that $\sigma\left(-I, A_{i}\right) \supseteq \sigma\left(-I, A_{i}^{\prime}\right)$ and $\sigma\left(-I, A_{i}, A_{j}\right) \supseteq \sigma\left(-I, A_{i}^{\prime}, A_{j}^{\prime}\right)$, hence also that $\sigma_{p}\left(A_{i}, A_{j}\right) \supseteq \sigma_{p}\left(A_{i}^{\prime}, A_{j}^{\prime}\right)$. In particular, for each $i$ we have $\{-1,1\}=\sigma\left(A_{i}\right) \supseteq \sigma\left(A_{i}^{\prime}\right)$. Since left regular representations are unitary, $A_{i}^{\prime}$ is normal and hence an involution by Proposition 3.1 .

Next, suppose that the Coxeter exponent $m_{i j}$ is finite. Then by Lemma 4.3 the spectrum of $A_{i} A_{j}+A_{j} A_{i}$ is finite and contained in the set $\left\{2 \cos \left(2 \pi k / m_{i j}\right) \mid k=\right.$ 
$\left.0, \ldots, m_{i j}-1\right\}$. Hence by Lemma 4.1 it is completely determined by $\sigma_{p}\left(A_{i}, A_{j}\right)$, which is a finite union of ellipses. Since $\sigma_{p}\left(A_{i}^{\prime}, A_{j}^{\prime}\right)$ is contained inside $\sigma_{p}\left(A_{i}, A_{j}\right)$, again Lemma 4.1 tells us that the spectrum of $A_{i}^{\prime} A_{j}^{\prime}+A_{j}^{\prime} A_{i}^{\prime}$ is finite and contained in the spectrum of $A_{i} A_{j}+A_{j} A_{i}$. Therefore by Lemma 4.3 we obtain $\left(A_{1}^{\prime} A_{2}^{\prime}\right)^{m_{i j}}=I$. Since the left regular representation is faithful, the generators $g_{i}^{\prime}$ of $G^{\prime}$ satisfy the defining relations on the generators $g_{i}$ of $G$.

Now suppose $G$ is finite and $D(T)=D\left(T^{\prime}\right)$ as subschemes of $\mathbb{C P}^{n}$. But then $|G|=\operatorname{deg} D(T)=\operatorname{deg} D\left(T^{\prime}\right)=\left|G^{\prime}\right|$, hence the epimorphism $f$ has to be an isomorphism.

\section{REPRESENTATIONS OF DIHEDRAL GROUPS}

We investigate finite dimensional unitary representations of dihedral groups.

The following result was for us the first indication that joint spectra can be employed to study Coxeter groups. Observe how the proof uses the equation of the circle to explicitly construct the desired invariant subspace.

Theorem 5.1. Let $A_{1}, A_{2}$ be self-adjoint linear operators on an $N$-dimensional Hilbert space $V$, and suppose that $A_{1}$ is invertible and that $\left\|A_{2}\right\|=1$. Further suppose that the "complex unit circle" $\left\{(x, y) \in \mathbb{C}^{2}: x^{2}+y^{2}=1\right\}$ is a reduced component of both $\sigma_{p}\left(A_{1}, A_{2}\right)$ and $\sigma_{p}\left(A_{1}^{-1}, A_{2}\right)$, of multiplicity $n$ in $\sigma_{p}\left(A_{1}, A_{2}\right)$, that the points $( \pm 1,0)$ do not belong to any other component of either $\sigma_{p}\left(A_{1}, A_{2}\right)$ or $\sigma_{p}\left(A_{1}^{-1}, A_{2}\right)$, and that the points $(0, \pm 1)$ do not belong to any other component of $\sigma_{p}\left(A_{1}, A_{2}\right)$. Then:

(1) $A_{1}$ and $A_{2}$ have a common $2 n$-dimensional invariant subspace $L$;

(2) The pair of restrictions $\left.A_{1}\right|_{L}$ and $\left.A_{2}\right|_{L}$ is unitary equivalent to the following pair of $2 n \times 2 n$ involutions $C_{1}$ and $C_{2}$, each block-diagonal with $n$ equal $2 \times 2$ blocks along the diagonal:

$$
C_{1}=\left[\begin{array}{ccccc}
1 & 0 & \ldots & 0 & 0 \\
0 & -1 & \ldots & 0 & 0 \\
\vdots & \vdots & \ddots & \vdots & \vdots \\
0 & 0 & \ldots & 1 & 0 \\
0 & 0 & \ldots & 0 & -1
\end{array}\right], C_{2}=\left[\begin{array}{ccccc}
0 & 1 & \ldots & 0 & 0 \\
1 & 0 & \ldots & 0 & 0 \\
\vdots & \vdots & \ddots & \vdots & \vdots \\
0 & 0 & \ldots & 0 & 1 \\
0 & 0 & \ldots & 1 & 0
\end{array}\right]
$$

(3) The group generated by $C_{1}$ and $C_{2}$ represents the Coxeter group $B_{2}$.

Proof. Since the coordinate axes are not tangent to the unit circle at any of the points $( \pm 1,0)$ and $(0, \pm 1)$, and these are regular points of the unit circle, Proposition 3.2 shows that both 1 and -1 are eigenvalues of $A_{1}$ and of $A_{2}$ with multiplicity $n$. Let $e_{1}, \ldots, e_{n}$ and $e_{n+1}, \ldots, e_{2 n}$ be a pair of orthonormal eigenbases for the eigenspaces of $A_{1}$ with eigenvalues 1 and -1 respectively, and $\xi_{1}, \ldots, \xi_{n}$ and $\xi_{n+1}, \ldots, \xi_{2 n}$ be a similar pair of orthonormal eigenbases for $A_{2}$. The spectral decomposition of $A_{1}$ in our case looks like

$$
A_{1}=P_{1}-P_{2}+\sum_{j=3}^{k} \lambda_{j} P_{j},
$$

where $P_{1}$ and $P_{2}$ are the orthogonal projections on the spaces $\operatorname{span}\left\{e_{1}, \ldots, e_{n}\right\}$ and $\operatorname{span}\left\{e_{n+1}, \ldots, e_{2 n}\right\}$ respectively, $\lambda_{j}$ for $j=3, \ldots, k$ are all other eigenvalues of $A_{1}$, 
and $P_{j}$ is the orthogonal projection on the $\lambda_{j}$-eigensubspace of $A_{1}$. In our situation equations (3.9) and (3.10) applied to the projection $P_{1}$ give

$$
\begin{array}{r}
P_{1} A_{2} P_{1}=0 \\
P_{1} A_{2} T A_{2} P_{1}=-\frac{1}{2} P_{1},
\end{array}
$$

where $T$ is given by (3.7), which applied to our case turns into

$$
T=-\frac{1}{2} P_{2}+\sum_{j=3}^{k} \frac{1}{\lambda_{j}-1} P_{j}
$$

Equations (5.2) and (5.3) imply that for every $j=1, \ldots, n$ we have

$$
\begin{aligned}
& P_{1} A_{2} T A_{2} e_{j}=-\frac{1}{2} e_{j}, \\
&-P_{1} A_{2}\left(\frac{1}{2} P_{2} A_{2} e_{j}+\sum_{s=3}^{k} \frac{1}{1-\lambda_{s}} P_{s} A_{2} e_{j}\right)=-\frac{1}{2} e_{j}, \\
& P_{1}\left(\frac{1}{2} A_{2} P_{2} A_{2} e_{j}+\sum_{s=3}^{k} \frac{1}{1-\lambda_{s}} A_{2} P_{s} A_{2} e_{j}\right)=\frac{1}{2} e_{j}, \\
& \sum_{l=1}^{n}\left[\frac{1}{2}\left\langle A_{2} P_{2} A_{2} e_{j}, e_{l}\right\rangle+\sum_{s=3}^{k} \frac{1}{1-\lambda_{s}}\left\langle A_{2} P_{s} A_{2} e_{j}, e_{l}\right\rangle\right] e_{l}=\frac{1}{2} e_{j}, \\
& \sum_{l=1}^{n}\left[\frac{1}{2}\left\langle P_{2} A_{2} e_{j}, A_{2} e_{l}\right\rangle+\sum_{s=3}^{k} \frac{1}{1-\lambda_{s}}\left\langle P_{s} A_{2} e_{j}, A_{2} e_{l}\right\rangle\right] e_{l}=\frac{1}{2} e_{j}, \\
& \sum_{l=1}^{n}\left[\frac{1}{2}\left\langle P_{2} A_{2} e_{j}, P_{2} A_{2} e_{l}\right\rangle+\sum_{s=3}^{k} \frac{1}{1-\lambda_{s}}\left\langle P_{s} A_{2} e_{j}, P_{s} A_{2} e_{l}\right\rangle\right] e_{l}=\frac{1}{2} e_{j},
\end{aligned}
$$

The last relation implies

$$
\frac{1}{2}\left\langle P_{2} A_{2} e_{j}, P_{2} A_{2} e_{l}\right\rangle+\sum_{s=3}^{k} \frac{1}{1-\lambda_{s}}\left\langle P_{s} A_{2} e_{j}, P_{s} A_{2} e_{l}\right\rangle=\frac{1}{2} \delta_{j}^{l}
$$

for all $1 \leq j, l \leq n$, where $\delta_{j}^{l}$ is the Kronecker symbol. Putting $l=j$ in (5.4) yields

$$
\frac{1}{2}\left|P_{2} A_{2} e_{j}\right|^{2}+\sum_{s=3}^{k} \frac{\left|P_{s}\left(A_{2} e_{j}\right)\right|^{2}}{1-\lambda_{s}}=\frac{1}{2}, \quad 1 \leq j \leq n .
$$

Since the spectral resolution for $A_{1}^{-1}$ is

$$
A_{1}^{-1}=P_{1}-P_{2}+\sum_{j=3}^{k} \frac{1}{\lambda_{j}} P_{j}
$$

the operator $T\left(A_{1}^{-1}\right)$ is given by

$$
T\left(A_{1}^{-1}\right)=-\frac{1}{2} P_{2}+\sum_{j=3}^{k} \frac{\lambda_{j}}{1-\lambda_{j}} P_{j}
$$


Now, equation (3.10) applied to $\sigma_{p}\left(A_{1}^{-1}, A_{2}\right)$ yields

$$
P_{1} A_{2} T\left(A_{1}^{-1}\right) A_{2} P_{1}=-\frac{1}{2} P_{1},
$$

A similar argument applied to (5.6) gives

$$
\frac{1}{2}\left|P_{2} A_{2} e_{j}\right|^{2}-\sum_{s=3}^{k} \frac{\lambda_{s}\left|P_{s} A_{2} e_{j}\right|^{2}}{1-\lambda_{s}}=\frac{1}{2} .
$$

Adding (5.7) and (5.5) we obtain

$$
\sum_{s=2}^{k}\left|P_{s} A_{2} e_{j}\right|^{2}=1
$$

Now, since (5.2) means $P_{1}\left(A_{2} e_{j}\right)=0$, the displayed equation above yields

$$
\left|A_{2} e_{j}\right|=1 \text {. }
$$

Since $\left\|A_{2}\right\|=1$, it follows from (5.9) that for every $1 \leq j \leq n$ we have $e_{j} \in$ $\operatorname{span}\left\{\xi_{1}, \ldots, \xi_{2 n}\right\}$. A similar analysis shows that for each $n+1 \leq j \leq 2 n$ we have $e_{j} \in \operatorname{span}\left\{\xi_{1}, \ldots, \xi_{2 n}\right\}$. Thus,

$$
L=\operatorname{span}\left\{\xi_{1}, \ldots, \xi_{2 n}\right\}=\operatorname{span}\left\{e_{1}, \ldots, e_{2 n}\right\}
$$

is a common invariant subspace of $A_{1}$ and $A_{2}$, which completes the proof of (1).

Next, we remark that the restrictions of $A_{1}$ and $A_{2}$ to $L$ are involutions, and, since $P_{1} A_{2} e_{j}=0$ for $j=1, \ldots, n$ and $P_{2} A_{2} e_{j}=0$ for $j=n+1, \ldots, 2 n$, that

$$
\begin{gathered}
A_{2} e_{j} \in \operatorname{span}\left\{e_{n+1}, \ldots, e_{2 n}\right\}, \text { for } j=1, \ldots, n ; \\
A_{2} e_{j} \in \operatorname{span}\left\{e_{1}, \ldots, e_{n}\right\}, \text { for } j=n+1, \ldots, 2 n .
\end{gathered}
$$

Therefore, in the basis $e_{1}, \ldots, e_{2 n}$ the restrictions of $A_{1}$ and $A_{2}$ to $L$ look like

$$
\left.A_{1}\right|_{L}=\left[\begin{array}{ll}
I_{n} & 0_{n} \\
0_{n} & -I_{n}
\end{array}\right],\left.\quad A_{2}\right|_{L}=\left[\begin{array}{ll}
0_{n} & D_{n} \\
D_{n}^{*} & 0_{n}
\end{array}\right],
$$

where $I_{n}$ and $0_{n}$ are the identity and zero $n \times n$ matrices respectively, and $D_{n}$ is a unitary $n \times n$ matrix. Write

$$
U=\left[\begin{array}{cc}
D_{n}^{*} & 0_{n} \\
0_{n} & I_{n}
\end{array}\right]
$$

then $U$ is unitary and

$$
U\left(\left[\begin{array}{cc}
I_{n} & 0_{n} \\
0_{n} & -I_{n}
\end{array}\right],\left[\begin{array}{cc}
0_{n} & D_{n} \\
D_{n}^{*} & 0_{n}
\end{array}\right]\right) U^{*}=\left(\left[\begin{array}{cc}
I_{n} & 0_{n} \\
0_{n} & -I_{n}
\end{array}\right],\left[\begin{array}{cc}
0_{n} & I_{n} \\
I_{n} & 0_{n}
\end{array}\right]\right) .
$$

Now, the interchange of coordinate vectors $e_{2 j} \leftrightarrow e_{2 j-1+n}$ for $j=1, \ldots,\left\lfloor\frac{n}{2}\right\rfloor$ finishes the proof of (2).

To prove (3) note that the relations $C_{1}^{2}=C_{2}^{2}=\left(C_{1} C_{2}\right)^{4}=1$ are straightforward to verify.

We now prove a result that builds upon Lemma 4.1 .

Theorem 5.14. Let $A_{1}$ and $A_{2}$ be unitary self-adjoint linear operators on a finitedimensional Hilbert space $V$. Then:

(1) Every reduced component of $\sigma_{p}\left(A_{1}, A_{2}\right)$ is either a line $\{x \pm y= \pm 1\}$ or an "ellipse" $\left\{x^{2}+2 x y \cos (2 \pi \theta)+y^{2}=1\right\}$ for some $0<\theta<1 / 2$. 
(2) If a line $\{x \pm y= \pm 1\}$ is a reduced component of multiplicity $r$ of the joint spectrum $\sigma_{p}\left(A_{1}, A_{2}\right)$ then $A_{1}$ and $A_{2}$ have a correponding common eigenspace of dimension $r$.

(3) If an "ellipse" $\left\{x^{2}+2 x y \cos (2 \pi \theta)+y^{2}=1\right\}$ with $0<\theta<1 / 2$ is a reduced component of the proper joint spectrum $\sigma_{p}\left(A_{1}, A_{2}\right)$ of multiplicity $r$, then $A_{1}$ and $A_{2}$ have a correponding common invariant subspace of dimension $2 r$ that is a direct sum of $r$ two-dimensional common invariant subspaces.

Proof. Since $A_{1}$ and $A_{2}$ are unitary involutions, the operator $R=A_{1} A_{2}+A_{2} A_{1}$ is self-adjoint of norm $\leq 2$. Part (1) now is an immediate consequence of Lemma 4.1 .

We give the proof of (2) for the case when our line is $\{x+y=1\}$. The proof of the other cases is analogous.

Choose a point $(\gamma, \beta) \in\{x+y=1\}$ with positive real coordinates. The spectrum of the operator $\gamma A_{1}+\beta A_{2}$ is the set $\left\{\lambda \in \mathbb{R}:(1 / \lambda)(\gamma, \beta) \in \sigma_{p}\left(A_{1}, A_{2}\right)\right\}$. Since by part (1) all nonlinear reduced components of $\sigma_{p}\left(A_{1}, A_{2}\right)$ are convex inside the positive real quadrant and intersect $\{x+y=1\}$ at $(1,0)$ and $(0,1)$, we have that $\max \left\{|\lambda|:(1 / \lambda)(\gamma, \beta) \in \sigma_{p}\left(A_{1}, A_{2}\right)\right\}$ corresponds to the point of the intersection of the lines $\{x+y=1\}$ and $\{\beta x-\gamma y=0\}$. Thus $\max \{|\lambda|:(1 / \lambda)(\gamma, \beta) \in$ $\left.\sigma_{p}\left(A_{1}, A_{2}\right)\right\}=1 /(\gamma+\beta)=1$. Since the operator $\gamma A_{1}+\beta A_{2}$ is self-adjoint, this implies $\left\|\gamma A_{1}+\beta A_{2}\right\|=1$. Also, this point of intersection is a regular point of $\sigma_{p}\left(A_{1}, A_{2}\right)$. By (3.4) the joint spectrum of $\gamma A_{1}+\beta A_{2}$ and $A_{2}$ also contains the line $\{x+y=1\}$ (with the same multiplicity $r$ ), and $(1,0)$ is a regular point of $\sigma_{p}\left(\gamma A_{1}+\beta A_{2}, A_{2}\right)$. Since $\left\|\gamma A_{1}+\beta A_{2}\right\|=\left\|A_{2}\right\|=1$, by [5, Proofs of Lemma 5 and Lemma 9] we have that $A_{2}$ and $\gamma A_{1}+\beta A_{2}$ have a common eigensubspace corresponding to the eigenvalue 1. By Proposition 3.2 this eigenspace has dimension equal to the multiplicity of the line $\{x+y=1\}$ in $\sigma_{p}\left(\gamma A_{1}+\beta A_{2}, A_{2}\right)$, hence is $r$-dimensional. Clearly it is an eigensubspace of eigenvalue 1 for $A_{1}$ as well.

Now we proceed with the proof of (3). In view of part (2), by restricting to orthogonal complements if necessary, we may assume without loss of generality that $\sigma_{p}\left(A_{1}, A_{2}\right)$ contains none of the lines $\{x \pm y=1\}$ or $\{x \pm y=-1\}$. By part (1) and its proof, all reduced components of the proper joint spectrum are the nonsingular "ellipses"

$$
\mathcal{E}_{i}=\left\{x^{2}+2 x y \cos \left(2 \pi \theta_{i}\right)+y^{2}=1\right\}, \text { with } 1 / 2>\theta_{1}>\cdots>\theta_{s}>0,
$$

their intersection points are $( \pm 1,0)$ and $(0, \pm 1)$, and $\alpha_{i}=2 \cos \left(2 \pi \theta_{i}\right)$ is an eigenvalue of $R=A_{1} A_{2}+A_{2} A_{1}$ for each $i$. Note that if $M_{i}$ is the corresponding eigenspace of $R$, then for every $(x, y) \in \mathbb{R}^{2}$ on the ellipse $\mathcal{E}_{i}$ the space $M_{i}$ is invariant under the action of $x A_{1}+y A_{2}$. Indeed, let $\xi \in M_{i}$. Then

$$
\left(x A_{1}+y A_{2}\right)^{2} \xi=\left(x^{2}+y^{2}\right) \xi+x y R_{1} \xi=\xi .
$$

Thus, the restriction of $\left(x A_{1}+y A_{2}\right)^{2}$ to $M_{i}$ is the identity of $M_{i}$. Since the eigenvalues of $\left(x A_{1}+y A_{2}\right)^{2}$ are just the squares of the eigenvalues of $x A_{1}+y A_{2}$ with the same eigenvectors, every eigenvector of $\left(x A_{1}+y A_{2}\right)^{2}$ with eigenvalue 1 is a linear combination of eigenvectors of $\left(x A_{1}+y A_{2}\right)$ with eigenvalues \pm 1 . Conversely, the displayed above equality shows also that when $x y \neq 0$ each eigenvector $\xi$ of $\left(x A_{1}+y A_{2}\right)$ with eigenvalue \pm 1 , is an eigenvector of $R$ with eigenvalue $\alpha_{i}$. Thus, when $x y \neq 0$ the eigenspace $M_{i}$ is the direct sum of the eigenspaces of $\left(x A_{1}+y A_{2}\right)$ with eigenvalues \pm 1 , and, therefore, is invariant under $\left(x A_{1}+y A_{2}\right)$. In particular, since when $x y \neq 0$ the point $(x, y)$ belongs to only one reduced component (of multiplicity $n_{i}$ ) of $\sigma_{p}\left(A_{1}, A_{2}\right)$, is a nonsingular point on that component, and no lines 
through the origin are tangent to any of the reduced components of $\sigma_{p}\left(A_{1}, A_{2}\right)$, by Proposition 3.2 the eigenspace of $x A_{1}+y A_{2}$ of eigenvalue 1 has dimension $n_{i}$. Since our components are invariant under the transformation $(x, y) \mapsto(-x,-y)$, when $x y \neq 0$ the eigenspace of $x A_{1}+y A_{2}$ of eigenvalue -1 is also of dimension $n_{i}$; hence the dimension of $M_{i}$ is $2 n_{i}$.

Next, the projective joint spectrum $\sigma\left(I, A_{1}, A_{2}\right)$ is the union of the homogenizations of the "ellipses" (5.15), with the same multiplicities. Furthermore, since there are no real solutions to the equation $x^{2}+2 x y \cos \left(2 \pi \theta_{i}\right)+y^{2}=0$, there are no real points of the form $[0: x: y]$ in $\sigma\left(I, A_{1}, A_{2}\right)$, i.e. no real points on the "infinite" line $\left\{x_{0}=0\right\}$; and hence 0 is not in the spectrum of $\left(x A_{1}+y A_{2}\right)$. Therefore for every $(x, y) \in \mathbb{R}^{2}$ with $(x, y) \neq(0,0)$ the operator $\left(x A_{1}+y A_{2}\right)$ is invertible. Also, if $(x, y) \in \mathbb{R}^{2}$ belongs to $\mathcal{E}_{i}$, then

$$
\left.\left(x A_{1}+y A_{2}\right)^{-1}\right|_{M_{i}}=\left.\left(x A_{1}+y A_{2}\right)\right|_{M_{i}} .
$$

Indeed, let $\xi \in M_{i}$. We have

$$
\left(x A_{1}+y A_{2}\right)^{2} \xi=\left(x^{2}+x y \alpha_{i}+y^{2}\right) \xi .
$$

Thus, $\left(x A_{1}+y A_{2}\right)^{-1}$ is defined by

$$
\left.\left(x A_{1}+y A_{2}\right)^{-1}\right|_{M_{i}}=\left.\frac{1}{x^{2}+x y \alpha_{i}+y^{2}}\left(x A_{1}+y A_{2}\right)\right|_{M_{i}}
$$

which, of course, implies (5.16).

Next we note that

$$
x^{2}+2 x y \cos \left(2 \pi \theta_{i}\right)+y^{2}=\frac{1+\cos \left(2 \pi \theta_{i}\right)}{2}(x+y)^{2}+\frac{1-\cos \left(2 \pi \theta_{i}\right)}{2}(x-y)^{2},
$$

and, therefore, each "ellipse" (5.15) is centered at the origin, has axes along lines $\{x=y\}$ and $\{x=-y\}$, the lengths of its semiaxes $\sqrt{\frac{1}{1+\cos \left(2 \pi \theta_{i}\right)}}$ and $\sqrt{\frac{1}{1-\cos \left(2 \pi \theta_{i}\right)}}$ respectively, and recall that we write $n_{i}$ for its multiplicity.

Set

$$
\begin{aligned}
& B_{1}=\frac{1}{\sqrt{2\left(1+\cos \left(2 \pi \theta_{1}\right)\right)}}\left(A_{1}+A_{2}\right) \\
& B_{2}=\frac{1}{\sqrt{2\left(1-\cos \left(2 \pi \theta_{1}\right)\right)}}\left(A_{1}-A_{2}\right) .
\end{aligned}
$$

It is easy to check that the joint spectrum of $B_{1}$ and $B_{2}$ is the union of the "ellipses"

$$
\mathcal{E}_{i}^{\prime}=\left\{\frac{1+\cos \left(2 \pi \theta_{i}\right)}{1+\cos \left(2 \pi \theta_{1}\right)} x^{2}+\frac{1-\cos \left(2 \pi \theta_{i}\right)}{1-\cos \left(2 \pi \theta_{1}\right)} y^{2}=1\right\}
$$

for $i=1, \ldots, s$, each with multiplicity $n_{i}$. In particular, this implies that the unit circle $\left\{x^{2}+y^{2}=1\right\}$ is in $\sigma_{p}\left(B_{1}, B_{2}\right)$, has multiplicity $n_{1}$, and the spectrum of $B_{2}$ consists of numbers $\left\{ \pm \sqrt{\frac{1-\cos \left(2 \pi \theta_{i}\right)}{1-\cos \left(2 \pi \theta_{1}\right)}}\right\}$. Hence, the norm of $B_{2}$ is equal to one. It also follows from (5.18) that the points $( \pm 1,0)$ and $(0, \pm 1)$ are regular points of $\sigma_{p}\left(B_{1}, B_{2}\right)$, all of them having multiplicity $n_{1}$. 
Finally, if $(x, y) \in \mathbb{R}^{2}$ belongs to $\mathcal{E}_{i}^{\prime}$, then

$$
\begin{aligned}
x B_{1}+ & y B_{2}=\left(\frac{1}{\sqrt{2\left(1+\cos \left(2 \pi \theta_{1}\right)\right)}} x+\frac{1}{\sqrt{2\left(1-\cos \left(2 \pi \theta_{1}\right)\right)}} y\right) A_{1} \\
& +\left(\frac{1}{\sqrt{2\left(1+\cos \left(2 \pi \theta_{1}\right)\right)}} x-\frac{1}{\sqrt{2\left(1-\cos \left(2 \pi \theta_{1}\right)\right)}} y\right) A_{2}=u_{1} A_{1}+u_{2} A_{2} .
\end{aligned}
$$

It is easy to check that

$$
u_{1}^{2}+2 u_{1} u_{2} \cos \left(2 \pi \theta_{i}\right)+u_{2}^{2}=1
$$

which, of course, means that $\left(u_{1}, u_{2}\right) \in \mathcal{E}_{i}$. According to (5.16)

$$
\begin{aligned}
\left.\left(x B_{1}+y B_{2}\right)^{-1}\right|_{M_{i}}=\left.\left(u_{1} A_{1}+u_{2} A_{2}\right)^{-1}\right|_{M_{i}} & \\
& =\left.\left(u_{1} A_{1}+u_{2} A_{2}\right)\right|_{M_{i}}=\left.\left(x B_{1}+y B_{2}\right)\right|_{M_{i}} .
\end{aligned}
$$

Let $\lambda_{i}=\sqrt{\frac{1+\cos \left(2 \pi \theta_{1}\right)}{1+\cos \left(2 \pi \theta_{i}\right)}}$. It follows that $\left.\left(\lambda_{i} B_{1}\right)^{-1}\right|_{M_{i}}=\left.\lambda_{i} B_{1}\right|_{M_{i}}$, hence

$$
\begin{aligned}
& \left.\left(x B_{1}^{-1}+y B_{2}\right)\right|_{M_{i}}=\left.x B_{1}^{-1}\right|_{M_{i}}+\left.y B_{2}\right|_{M_{i}} \\
& \quad=\left.x \lambda_{i}^{2} B_{1}\right|_{M_{1}}+\left.y B_{2}\right|_{M_{i}}=\left.\left(x \lambda_{i}^{2} B_{1}+y B_{2}\right)\right|_{M_{i}} .
\end{aligned}
$$

Therefore, except for finitely many points, when $\left(\lambda_{i}^{2} x, y\right)$ is on $\mathcal{E}_{i}^{\prime}$ the space $M_{i}$ is the direct sum of eigenspaces of eigenvalues \pm 1 for the operator $\left(x B_{1}^{-1}+y B_{2}\right)$. Thus each ellipse $\mathcal{E}_{i}^{\prime \prime}=\left\{\frac{1+\cos \left(2 \pi \theta_{1}\right)}{1+\cos \left(2 \pi \theta_{i}\right)} x^{2}+\frac{1-\cos \left(2 \pi \theta_{i}\right)}{1-\cos \left(2 \pi \theta_{1}\right)} y^{2}=1\right\}$ is a reduced component of $\sigma_{p}\left(B_{1}^{-1}, B_{2}\right)$, and by Proposition 3.2 it has multiplicity $\geq n_{i}$. Since the sum of the multiplicities equals $(1 / 2) \operatorname{dim} V$, we must have that the multiplicity of $\mathcal{E}_{i}^{\prime \prime}$ equals $n_{i}$ for each $i$, and therefore these are all reduced components of $\sigma_{p}\left(B_{1}^{-1}, B_{2}\right)$. In particular, the "unit circle" $\left\{x^{2}+y^{2}=1\right\}$ is a reduced component of $\sigma_{p}\left(B_{1}^{-1}, B_{2}\right)$, of multiplicity $n_{1}$, and the points $( \pm 1,0)$ do not belong to any other of its components. It follows from Theorem [5.1 that $M_{1}$ is a direct sum of $n_{1}$ two-dimensional common invariant subspaces for $B_{1}$ and $B_{2}$ and the restriction of the pair $B_{1}, B_{2}$ to each of these subspaces is unitary equivalent to the pair

$$
\left[\begin{array}{rr}
1 & 0 \\
0 & -1
\end{array}\right],\left[\begin{array}{ll}
0 & 1 \\
1 & 0
\end{array}\right]
$$

Of course, each of these subspaces is also invariant under $A_{1}$ and $A_{2}$, and the restrictions on these subspaces of the pair $A_{1}, A_{2}$ is unitary equivalent to

$$
\left[\begin{array}{rr}
\cos \left(\pi \theta_{1}\right) & \sin \left(\pi \theta_{1}\right) \\
\sin \left(\pi \theta_{1}\right) & -\cos \left(\pi \theta_{1}\right)
\end{array}\right],\left[\begin{array}{rr}
\cos \left(\pi \theta_{1}\right) & -\sin \left(\pi \theta_{1}\right) \\
-\sin \left(\pi \theta_{1}\right) & -\cos \left(\pi \theta_{1}\right)
\end{array}\right] .
$$

Denote by $\mathcal{N}_{1}$ the orthocomplement to $M_{1}$. Then $\mathcal{N}_{1}$ is invariant under $A_{1}$ and $A_{2}$ and the proper joint spectrum $\sigma_{p}\left(\left.A_{1}\right|_{\mathcal{N}_{1}},\left.A_{2}\right|_{\mathcal{N}_{1}}\right)$ is the union of the ellipses $\mathcal{E}_{j}$ with $j=2, \ldots, s$, each with multiplicity $n_{j}$. A similar argument applied to the subspace $M_{2}$ of $\mathcal{N}_{1}$ shows that $M_{2}$ is a direct sum of $n_{2}$ two-dimensional common invariant subspaces for $A_{1}$ and $A_{2}$, and that their restrictions to those subspaces are unitary equivalent to

$$
\left[\begin{array}{rr}
\cos \left(\pi \theta_{2}\right) & \sin \left(\pi \theta_{2}\right) \\
\sin \left(\pi \theta_{2}\right) & -\cos \left(\pi \theta_{2}\right)
\end{array}\right],\left[\begin{array}{rr}
\cos \left(\pi \theta_{2}\right) & -\sin \left(\pi \theta_{2}\right) \\
-\sin \left(\pi \theta_{2}\right) & -\cos \left(\pi \theta_{2}\right)
\end{array}\right] .
$$


Since $\theta=\theta_{j}$ for some $j$, iterating the above procedure $j$ times yields the claimed result.

As an immediate consequence of Theorem 5.14 and its proof we have:

Theorem 5.20. Let $G$ be a Coxeter group of type $I(n)$ for some $2 \leq n \leq \infty$, let $\left\{g_{1}, g_{2}\right\}$ be a set of Coxeter generators, and let $\rho: G \rightarrow G L(V)$ be a finite dimensional unitary representation of $G$.

(1) If $\rho^{\prime}: G \rightarrow G L\left(V^{\prime}\right)$ is another finite dimensional uintary representation of $G$ such that $\sigma_{p}\left(\rho\left(g_{1}\right), \rho\left(g_{2}\right)\right)=\sigma_{p}\left(\rho^{\prime}\left(g_{1}\right), \rho^{\prime}\left(g_{2}\right)\right)$ as subschemes of $\mathbb{C}^{2}$, then $\rho$ and $\rho^{\prime}$ are equivalent.

(2) If $\rho$ is irreducible then $\sigma_{p}\left(\rho\left(g_{1}\right), \rho\left(g_{2}\right)\right)$ is reduced and irreducible.

\section{Admissible transformations}

For the rest of this paper $n \geq 2$, the group $G$ is a finite Coxeter group of type $A_{n}, B_{n}$, or $D_{n+1}$, and $\left\{g_{1}, g_{2}, \ldots\right\}$ is a set of Coxeter generators for $G$.

For a word $w=g_{i_{1}} \ldots g_{i_{N}}$, where the same letter $g_{k}$ might occur more than once, we write $|w|=N$. Let $a_{k}$ be the number of times $g_{k}$ occurs in $w$. The signature of $w$ is the sequence

$$
\operatorname{sig}(w)=\left(a_{1}, a_{2}, \ldots\right),
$$

and the content of $w$ is the sequence

$$
\operatorname{ct}(w)=\left(|w|, a_{1}, \cdots, a_{n-1}\right) \in \mathbb{N}^{n} .
$$

Since $|w|=a_{1}+a_{2}+\ldots$, in types $A_{n}$ and $B_{n}$ the content and the signature of $w$ carry the same information, while in type $D_{n+1}$ it is clearly possible to have words with the same content but different signatures. Let $\leq_{l e x}$ be the lexicographic ordering on $\mathbb{N}^{n}$, that is, $c=\left(c_{0}, \ldots, c_{n-1}\right)<_{\text {lex }}\left(d_{0}, \ldots, d_{n-1}\right)=d$ precisely when $c \neq d$ and $c_{m}<d_{m}$ for $m=\min \left\{i \mid c_{i} \neq d_{i}\right\}$. We always consider our words partially ordered $<$ by

$$
w_{1}<w_{2} \text { if and only if } \operatorname{ct}\left(w_{1}\right)<_{\text {lex }} \operatorname{ct}\left(w_{2}\right) .
$$

For example, $g_{2} g_{1} g_{2}<g_{1} g_{2} g_{1}$ whenever $n \geq 2$.

Definition 6.1. (a) We introduce admissible transformations on words. They are:

(1) Cancelling transformations. That is, for each $i$ the transformation

$$
w^{\prime} g_{i} g_{i} w^{\prime \prime} \mapsto w^{\prime} w^{\prime \prime}
$$

is admissible.

(2) Commuting transformations. This means that if $g_{i}$ and $g_{j}$ commute, then the transformation

$$
w^{\prime} g_{i} g_{j} w^{\prime \prime} \mapsto w^{\prime} g_{j} g_{i} w^{\prime \prime}
$$

is admissible.

(3) Circular transformations. Those are:

$$
g_{i_{1}} \ldots g_{i_{k}} g_{i_{k+1}} \ldots g_{i_{N}} \mapsto g_{i_{k+1}} \ldots g_{i_{N}} g_{i_{1}} \ldots g_{i_{k}} .
$$


(4) Replacement transformations. These replace a certain subword consisting of a successive string of letters by another representation of this subword. Specifically,

$$
\begin{aligned}
w^{\prime} g_{i} g_{i+1} g_{i} w^{\prime \prime} & \mapsto w^{\prime} g_{i+1} g_{i} g_{i+1} w^{\prime \prime} & & \text { for } 1 \leq i \leq n-2 ; \\
w^{\prime} g_{n-1} g_{n} g_{n-1} w^{\prime \prime} & \mapsto w^{\prime} g_{n} g_{n-1} g_{n} w^{\prime \prime} & & \text { in } A_{n} \text { and } D_{n+1} ; \\
w^{\prime} g_{n-1} g_{n+1} g_{n-1} w^{\prime \prime} & \mapsto w^{\prime} g_{n+1} g_{n-1} g_{n+1} w^{\prime \prime} & & \text { in } D_{n+1} ;
\end{aligned}
$$

and in addition the "tent commuting" replacements

$$
\begin{aligned}
& w^{\prime} t_{k} g_{j} w^{\prime \prime} \mapsto w^{\prime} g_{j} t_{k} w^{\prime \prime} \\
& \quad \text { for } k<j \leq n-1 \text { in } B_{n} \text { and } D_{n+1} ; \\
& w^{\prime} t_{k} g_{n} w^{\prime \prime} \mapsto w^{\prime} g_{n+1} t_{k} w^{\prime \prime} \text { and } \\
& w^{\prime} t_{k} g_{n+1} w^{\prime \prime} \mapsto w^{\prime} g_{n} t_{k} w^{\prime \prime} \\
& \quad \text { for } k \leq n-1 \text { in } D_{n+1} ; \\
& w^{\prime} t_{k} g_{n} w^{\prime \prime} \mapsto w^{\prime} g_{n} t_{k} w^{\prime \prime} \\
& \text { for } k \leq n-1 \text { in } B_{n}
\end{aligned}
$$

are admissible transformations. Here the $k$ th "tent word" $t_{k}$ is defined for $1 \leq k \leq n-1$ as

$$
t_{k}= \begin{cases}g_{k} \ldots g_{n-1} g_{n} g_{n-1} \ldots g_{k} & \text { in } B_{n} \\ g_{k} \ldots g_{n-1} g_{n} g_{n+1} g_{n-1} \ldots g_{k} & \text { in } D_{n+1}\end{cases}
$$

(b) We write $w_{1} \leadsto w_{2}$ if there is a sequence of admissible transformations that maps $w_{1}$ into $w_{2}$. Taking the equivalence closure of this transitive relation on words, we obtain the notion of $c$-equivalence. We write $w_{1} \sim w_{2}$ if the words $w_{1}$ and $w_{2}$ are $c$-equivalent.

Remark 6.2. (a) Note that in $B_{n}$ and $D_{n+1}$ we have the following "tent commuting" equalities of elements. First,

$$
\begin{aligned}
t_{k} g_{j} & =u g_{j-1} g_{j} t_{j+1} g_{j} g_{j-1}\left(v g_{j}\right) \\
& =u g_{j-1} g_{j} t_{j+1}\left(g_{j} g_{j-1} g_{j}\right) v \\
& =u g_{j-1} g_{j}\left(t_{j+1} g_{j-1}\right) g_{j} g_{j-1} v \\
& =u\left(g_{j-1} g_{j} g_{j-1}\right) t_{j+1} g_{j} g_{j-1} v \\
& =\left(u g_{j}\right) g_{j-1} g_{j} t_{j+1} g_{j} g_{j-1} v \\
& =g_{j}\left(u g_{j-1} g_{j} t_{j+1} g_{j} g_{j-1} v\right) \\
& =g_{j} t_{k}
\end{aligned}
$$

whenever $k<j \leq n-1$, as $u$ and $v$ only involve letters $g_{i}$ with $i \leq j-2$ hence they commute with $g_{j}$, while $t_{j+1}$ only involves $g_{i}$ s with $i \geq j+1$, hence it commutes with $g_{j-1}$. For trivial reasons, the same equality also holds when $j+1<k \leq n-1$. 
In a similar manner, when $k \leq n-1$ we have in $D_{n+1}$ the equalities

$$
\begin{aligned}
t_{k} g_{n} & =u g_{n-1}\left(g_{n} g_{n+1}\right) g_{n-1}\left(v g_{n}\right) \\
& =u g_{n-1} g_{n+1}\left(g_{n} g_{n-1} g_{n}\right) v \\
& =u\left(g_{n-1} g_{n+1} g_{n-1}\right) g_{n} g_{n-1} v \\
& =\left(u g_{n+1}\right) g_{n-1}\left(g_{n+1} g_{n}\right) g_{n-1} v \\
& =g_{n+1}\left(u g_{n-1} g_{n} g_{n+1} g_{n-1} v\right) \\
& =g_{n+1} t_{k}
\end{aligned}
$$

and $t_{k} g_{n+1}=g_{n} t_{k}$. Finally, in $B_{n}$ we have the equalities

$$
\begin{aligned}
t_{k} g_{n} & =u g_{n-1} g_{n} g_{n-1}\left(v g_{n}\right) \\
& =u\left(g_{n-1} g_{n} g_{n-1} g_{n}\right) v \\
& =\left(u g_{n}\right) g_{n-1} g_{n} g_{n-1} v \\
& =g_{n}\left(u g_{n-1} g_{n} g_{n-1} v\right) \\
& =g_{n} t_{k}
\end{aligned}
$$

for all $k \leq n-1$.

(b) Combining the above yields in $B_{n}$ and $D_{n+1}$ also the tent commuting equalities

whenever $1 \leq k, j \leq n-1$.

$$
t_{k} t_{j}=t_{j} t_{k}
$$

(c) It is immediate from (a) and the defining relations of $G$ that if $w_{1} \leadsto w_{2}$ and no circular transformations were used, then $w_{1}$ and $w_{2}$ represent the same element of the group $G$.

(d) It is also clear from (a) that if $w_{1}$ is $c$-equivalent to $w_{2}$ then these words represent elements of $G$ that belong to the same conjugacy class. The converse to this is also true, but since we will not need it here, we leave the easy though somewhat cumbersome proof as an exercise for the interested reader.

We conclude this section with a simple but crucially important observation.

Lemma 6.3. If $w_{1} \leadsto w_{2}$ then $\operatorname{ct}\left(w_{1}\right) \geq_{\text {lex }} \operatorname{ct}\left(w_{2}\right)$.

Proof. Indeed every listed admissible transformation either preserves the content of the word, or lowers it.

\section{ECHELON FORMS}

Now we will show that words in our Coxeter group $G$ have conjugates in a special form that we call echelon form.

Definition 7.1. We say that a word $w$ is in echelon form if it is of the form

$$
w=\delta_{1} \delta_{2} \ldots \delta_{n} \delta_{n+1}
$$

where for each $i$ the word $\delta_{i}$ satisfies

$$
\delta_{i} \in \begin{cases}\left\{1, g_{i}\right\} & \text { if } i \leq n \text { and } G=A_{n} ; \\ \{1\} & \text { if } i=n+1 \text { and } G=A_{n} \text { or } G=B_{n} ; \\ \left\{1, g_{n}\right\} & \text { if } i=n \text { and } G=B_{n} ; \\ \left\{1, g_{i}\right\} & \text { if } i \geq n \text { and } G=D_{n+1} ; \\ \left\{1, g_{i}, t_{i}\right\} & \text { if } i \leq n-1 \text { and } G=B_{n} \text { or } G=D_{n+1} .\end{cases}
$$


Remark 7.3. Note that when $w$ is in echelon form, it can be recovered from $\operatorname{ct}(w)$ in types $A_{n}$ and $B_{n}$, while in type $D_{n+1}$ there are at most two possibilities for $w$ given its content. More precisely, in all types, given $\operatorname{ct}(w)=\left(|w|, a_{1}, \ldots, a_{n-1}\right)$, the tuple $\left(a_{1}, \ldots, a_{i}\right)$ determines the tuple $\left(\delta_{1}, \ldots, \delta_{i}\right)$ whenever $1 \leq i \leq n-1$. In types $A_{n}$ and $B_{n}$ the tuple $\left(\delta_{1}, \ldots, \delta_{n-1}\right)$ together with $|w|$ determines $\delta_{n}$ and hence $w$. In type $D_{n+1}$ the tuple $\left(\delta_{1}, \ldots, \delta_{n-1}\right)$ together with $|w|$ leaves at most two possibilities for $\left(\delta_{n}, \delta_{n+1}\right)$, depending on the parity of $a_{n}+a_{n+1}$. If this parity is even, there is no choice. If the parity is odd, then there is a choice between either $\left(g_{n}, 1\right)$ or $\left(1, g_{n+1}\right)$ for $\left(\delta_{n}, \delta_{n+1}\right)$.

We can extend the last remark even further.

Proposition 7.4. Let $w_{1}$ and $w_{2}$ be two words in $D_{n+1}$, both in echelon form, and suppose that $\operatorname{ct}\left(w_{1}\right)=\operatorname{ct}\left(w_{2}\right)=\left(W, a_{1}, \ldots, a_{n-1}\right)$.

If $W-a_{1}-\cdots-a_{n-1} \geq 3$ then $w_{1}$ and $w_{2}$ belong to the same conjugacy class as elements of $G$.

Proof. By Remark 7.3 we may assume that $a_{n}+a_{n+1}=W-a_{1}-\cdots-a_{n-1}$ is odd and that

$$
w_{1}=\delta_{1} \ldots \delta_{n-1} g_{n} \quad \text { and } \quad w_{2}=\delta_{1} \ldots \delta_{n-1} g_{n+1},
$$

where each $\delta_{i}$ satisfies (7.2). Since $a_{n}+a_{n+1} \geq 3$, we must have $\delta_{i}=t_{i}$ for at least one index $i$. Let $k$ be the biggest such index, and let $j$ be the second biggest (if such exists). Then $\delta_{i} \in\left\{1, g_{i}\right\}$ for each $j<i<n$ with $i \neq k$, hence the tent commuting equalities from Remark 6.2 yield

$$
\begin{aligned}
w_{1} & =\delta_{1} \ldots \delta_{j-1} t_{j}\left(\delta_{j+1} \ldots \delta_{k-1}\right) t_{k}\left(\delta_{k+1} \ldots \delta_{n-1}\right) g_{n} \\
& =\delta_{1} \ldots \delta_{j-1} t_{j}\left(\delta_{k+1} \ldots \delta_{n-1}\right)\left(\delta_{j+1} \ldots \delta_{k-1}\right) t_{k} g_{n} \\
& =\delta_{1} \ldots \delta_{j-1} t_{j}\left(\delta_{k+1} \ldots \delta_{n-1}\right)\left(\delta_{j+1} \ldots \delta_{k-1}\right) g_{n+1} t_{k} \\
& =\delta_{1} \ldots \delta_{j-1} t_{j}\left(\delta_{k+1} \ldots \delta_{n-1}\right) g_{n+1}\left(\delta_{j+1} \ldots \delta_{k-1}\right) t_{k} .
\end{aligned}
$$

Now applying a circular transformation we obtain

$$
\sim\left(\delta_{j+1} \ldots \delta_{k-1}\right) t_{k} \delta_{1} \ldots \delta_{j-1} t_{j}\left(\delta_{k+1} \ldots \delta_{n-1}\right) g_{n+1}
$$

and using again the tent commuting equalities we get

$$
=\delta_{1} \ldots \delta_{j-1} t_{j}\left(\delta_{j+1} \ldots \delta_{k-1}\right) t_{k}\left(\delta_{k+1} \ldots \delta_{n-1}\right) g_{n+1}=w_{2}
$$

as desired.

The following key theorem is the main result of this section.

Theorem 7.5 (Ordering Theorem). Let $w$ be a word. There exists a word $\tilde{w}$ in echelon form such that $w \leadsto \tilde{w}$.

The proof relies on the following lemma.

Lemma 7.6. Let $i$ be the smallest index of a letter in $w$. Then $w \leadsto w^{\prime} \delta_{i} w^{\prime \prime}$ where the words $w^{\prime}$ and $w^{\prime \prime}$ can contain only letters with index $>i$, and $\delta_{i}$ satisfies (7.2). Furthermore, the sequence of admissible transformations can be chosen so that no circular transformations are used.

Proof. The case when $i \geq n$ is trivial, so we may assume that $i \leq n-1$, hence without loss of generality also that $i=1$. If $a_{1}=1$ then no transformations are 
needed, so we may assume that $a_{1} \geq 2$. Thus we have $w=u g_{1} v g_{1} s$ where $u$ and $v$ do not involve $g_{1}$.

Consider now the case $n=2$. We prove this case by induction on $a_{1}$. Suppose first that $a_{1}=2$. Then $s$ does not involve $g_{1}$. Since $v$ can involve only $g_{2}$ and/or $g_{3}$, a sequence of commuting and/or cancelling transformations yields $w \leadsto u g_{1} g_{2} g_{1} s$ or, in case of $D_{3}$, either $w \leadsto u g_{1} g_{2} g_{3} g_{1} s$ or $w \leadsto u g_{1} g_{3} g_{1} s$. When $G=A_{2}$ or $G=D_{3}$ a replacement transformation gives $u g_{1} g_{2} g_{1} s \leadsto u g_{2} g_{1} g_{2} s$ which is in the desired form. When $G=B_{2}$ we already have $u g_{1} g_{2} g_{1} s=u t_{1} s$ is in the desired form. When $G=D_{3}$ then $u g_{1} g_{2} g_{3} g_{1} s=u t_{1} s$ is also in the desired form, and a replacement transformation yields $u g_{1} g_{3} g_{1} s \leadsto u g_{3} g_{1} g_{3} s$ which is also in the desired form. Suppose next that $a_{1} \geq 3$. Then $s=s^{\prime} g_{1} s^{\prime \prime}$ where $s^{\prime \prime}$ does not involve $g_{1}$. By induction we have $u g_{1} v g_{1} s^{\prime} \sim u^{\prime} \delta_{1} v^{\prime}$ where $u^{\prime}$ and $v^{\prime}$ do not involve $g_{1}$ and no circular transformations were used, hence the same sequence of transformations yields $w \leadsto u^{\prime} \delta_{1} v^{\prime} g_{1} s^{\prime \prime}$. Since $v^{\prime}$ can involve only $g_{2}$ and/or $g_{3}$, a sequence of cancelling and/or commuting transformations yields $w \leadsto u^{\prime} \delta_{1} g_{2} g_{1} s^{\prime \prime}$ or, in case of $D_{3}$, also either $w \leadsto u^{\prime} \delta_{1} g_{2} g_{3} g_{1} s^{\prime \prime}$ or $w \leadsto u^{\prime} \delta_{1} g_{3} g_{1} s^{\prime \prime}$. In case $A_{2}$ we have $\delta_{1}=g_{1}$ hence a replacement transformation brings us to $w \leadsto u^{\prime} g_{2} g_{1} g_{2} s^{\prime \prime}$ which is in the desired form. In case $B_{2}$ and $\delta_{1}=g_{1}$ then we are already have $u^{\prime} g_{1} g_{2} g_{1} s^{\prime \prime}=u^{\prime} t_{1} s^{\prime \prime}$ which is in the desired form. In case $B_{2}$ and $\delta_{1}=t_{1}=g_{1} g_{2} g_{1}$ then a tent commuting transformation followed by cancelling yields $u^{\prime} t_{1} g_{2} g_{1} s^{\prime \prime} \leadsto$ $u^{\prime} g_{2} t_{1} g_{1} s^{\prime \prime}=u^{\prime} g_{2} g_{1} g_{2} g_{1} g_{1} s^{\prime \prime} \leadsto u^{\prime} g_{2} g_{1} g_{2} s^{\prime \prime}$ which is again in the desired form. In the case $D_{3}$ and $\delta_{1}=g_{1}$ we handle the possibilities $u^{\prime} g_{1} g_{2} g_{1} s^{\prime \prime}$ and $u^{\prime} g_{1} g_{3} g_{1} s^{\prime \prime}$ just as in the case $A_{2}$, and the possibility $u^{\prime} g_{1} g_{2} g_{3} g_{1} s^{\prime \prime}=u^{\prime} t_{1} s^{\prime \prime}$ is already in the desired form. Finally, we look at the case $D_{3}$ and $\delta_{1}=t_{1}=g_{1} g_{2} g_{3} g_{1}$. Then using tent commuting transformations yields $u^{\prime} t_{1} v^{\prime} g_{1} s^{\prime \prime} \leadsto u^{\prime} v^{\prime \prime} t_{1} g_{1} s^{\prime \prime}$ where $v^{\prime \prime}$ is obtained by exchanging the letters $g_{2}$ and $g_{3}$ in $v^{\prime}$. Then, a cancelling transformation produces $u^{\prime} v^{\prime \prime} t_{1} g_{1} s^{\prime \prime}=u^{\prime} v^{\prime \prime} g_{1} g_{2} g_{3} g_{1} g_{1} s^{\prime \prime} \leadsto u^{\prime} v^{\prime \prime} g_{1} g_{2} g_{3} s^{\prime \prime}$ which is again in the desired form and completes the proof of the case $n=2$.

Next, suppose $n \geq 3$. By induction on $n$ we have that $v \sim v^{\prime} \delta_{2} v^{\prime \prime}$ where $v^{\prime}$ and $v^{\prime \prime}$ can only involve letters with index 3 or higher, and no circular transformations are used. Therefore the same sequence of admissible transformations yields $w \leadsto$ $u g_{1} v^{\prime} \delta_{2} v^{\prime \prime} g_{1} t$. Since $g_{1}$ commutes with all the letters appearing in $v^{\prime}$ and $v^{\prime \prime}$, a sequence of commuting transformations yields $w \leadsto u v^{\prime} g_{1} \delta_{2} g_{1} v^{\prime \prime} t$. If $\delta_{2}=1$ then a cancelling transformation yields $w \leadsto u v^{\prime} v^{\prime \prime} t$; if $\delta_{2}=g_{2}$ then a replacement transformation gives $w \leadsto u v^{\prime} g_{2} g_{1} g_{2} v^{\prime \prime} t$; and if $\delta_{2}=t_{2}$ then we have $u v^{\prime} g_{1} t_{2} g_{1} v^{\prime \prime} t=$ $u v^{\prime} t_{1} v^{\prime \prime} t$. If $a_{1}=2$ then all three cases above are in the desired form. If $a_{1} \geq 3$ then the first two cases have a smaller $a_{1}$ and in them we are done by induction on $a_{1}$. In the last case, induction on $a_{1}$ gives $v^{\prime \prime} t \leadsto z^{\prime} \delta_{1} z^{\prime \prime}$ where $z^{\prime}$ and $z^{\prime \prime}$ do not involve $g_{1}$, and no circular transformations are used. Therefore the same sequence of transformations yields $w \leadsto u v^{\prime} t_{1} z^{\prime} \delta_{1} z^{\prime \prime}$, hence a sequence of tent commuting replacement transformations produces $w \leadsto u^{\prime \prime} t_{1} \delta_{1} z^{\prime \prime}$. Now, if $\delta_{1}=g_{1}$ then $t_{1} \delta_{1}=$ $g_{1} t_{2} g_{1} g_{1}$ hence a cancelling transformation yields $w \leadsto u^{\prime \prime} g_{1} t_{2} z^{\prime \prime}$ which is in the desired form. Finally, if $\delta_{1}=t_{1}$ we get $t_{1} \delta_{1}=g_{1} t_{2} g_{1} g_{1} t_{2} g_{1}$ hence a cancelling transformation yields $w \leadsto u^{\prime \prime} g_{1} t_{2} t_{2} g_{1} z^{\prime \prime}$ which has smaller $a_{1}$ hence we are done by induction on $a_{1}$ in this case as well. This completes the proof of the lemma.

Proof of Theorem 7.5. Let $i_{1}$ be the smallest index of a letter in $w$. By Lemma 7.6 we get $w \leadsto w^{\prime} \delta_{i_{1}} w^{\prime \prime}$ hence a circular trasnformation produces $w \leadsto \delta_{i_{1}} w^{\prime \prime} w^{\prime}=$ $\delta_{1} \ldots \delta_{i_{1}} w_{i_{1}}$ where $w_{i_{1}}$ only involves letters with index $>i_{1}$. Suppose for some 
$k \leq n-1$ we have already obtained $w \leadsto \delta_{1} \ldots \delta_{k} w_{k}$ where $w_{k}$ can only involve letters with index $>k$. If $k=n-1$ then the only possible letters involved in $w_{n-1}$ are $g_{n}$ and $g_{n+1}$, so we are done after a sequence of commuting and/or cancelling transformations. If $k<n-1$ then by Lemma 7.6 we have $w_{k} \leadsto w_{k}^{\prime} \delta_{k+1} w_{k}^{\prime \prime}$ where no circular transformations are used, and $w_{k}^{\prime}$ and $w_{k}^{\prime \prime}$ can involve only letters with index $>k+1$. Thus the same sequence of transformations yields $w \leadsto \delta_{1} \ldots \delta_{k} w_{k}^{\prime} \delta_{k+1} w_{k}^{\prime \prime}$. Therefore a sequence of commuting and/or tent commuting transformations produces $w \leadsto z \delta_{1} \ldots \delta_{k+1} w_{k}^{\prime \prime}$ where $z$ can involve only letters with index $>k+1$. Now a circular transformation gives $w \leadsto \delta_{1} \ldots \delta_{k+1} w_{k+1}$ where $w_{k+1}=w_{k}^{\prime \prime} z$ can involve only letters of index $>k+1$. Iterating this process we arrive at the desired conclusion.

\section{The Proof of Theorem 1.2}

We are now ready to present the proof of our second main result.

Proof of Theorem 1.2. By Theorem 2.1 we may assume that both $\rho_{1}$ and $\rho_{2}$ are unitary. Also, the equality $D\left(T, \rho_{1}\right)=D\left(T, \rho_{2}\right)$ implies $\operatorname{dim} V_{1}=\operatorname{deg} D\left(T, \rho_{1}\right)=$ $\operatorname{deg} D\left(T, \rho_{2}\right)=\operatorname{dim} V_{2}$, and

$$
\sigma_{p}\left(\rho_{1}\left(g_{1}\right), \ldots, \rho_{1}\left(g_{n}\right)\right)=\sigma_{p}\left(\rho_{2}\left(g_{1}\right), \ldots, \rho_{2}\left(g_{n}\right)\right) .
$$

In particular, the case when $G$ is finite of type $I(n)$ is an immediate consequence from Theorem 5.20 .

In the remaining cases for $G$ it suffices by Theorem 2.2 to show that the equality of subschemes $D\left(T, \rho_{1}\right)=D\left(T, \rho_{2}\right)$ implies that the characters $\chi_{\rho_{1}}$ and $\chi_{\rho_{2}}$ are the same.

Let $N=\operatorname{dim} V_{1}=\operatorname{dim} V_{2}$. For each $i$ let $A_{i}=\rho_{1}\left(g_{i}\right)$ and let $B_{i}=\rho_{2}\left(g_{i}\right)$. For every $\left(x_{1}, \ldots, x_{n}\right) \in \mathbb{C}^{n}$ the characteristic polynomials for the operators $x_{1} A_{1}+\cdots+$ $x_{n} A_{n}$ and $x_{1} B_{1}+\cdots+x_{n} B_{n}$ are equal. Therefore the spectra of $x_{1} A_{1}+\cdots+x_{n} A_{n}$ and $x_{1} B_{1}+\cdots+x_{n} B_{n}$ are the same counting multiplicities. This implies that the traces of these operators are the same, in other words

$$
x_{1} \operatorname{Tr}\left(A_{1}\right)+\cdots+x_{n} \operatorname{Tr}\left(A_{n}\right)=x_{1} \operatorname{Tr}\left(B_{1}\right)+\cdots+x_{n} \operatorname{Tr}\left(B_{n}\right) .
$$

Since this is true for all $x_{1}, \ldots, x_{n}$ we obtain

$$
\operatorname{Tr}\left(A_{j}\right)=\operatorname{Tr}\left(B_{j}\right), j=1, \ldots, n .
$$

Similarly, since for each $m$ the eigenvalues of $\left(x_{1} A_{1}+\cdots+x_{n} A_{n}\right)^{m}$ are just the $m$ th powers of the eigenvalues of $x_{1} A_{1}+\cdots+x_{n} A_{n}$, we get that for every $m \in \mathbb{N}$ the spectra of $\left(x_{1} A_{1}+\cdots+x_{n} A_{n}\right)^{m}$ and $\left(x_{1} B_{1}+\cdots+x_{n} B_{n}\right)^{m}$ are the same counting multiplicities. Since this is true for arbitrary $\left(x_{1}, \ldots, x_{n}\right) \in \mathbb{C}^{n}$, we obtain that for every $m$ and $\alpha=\left(a_{1}, \ldots, a_{n}\right) \in \mathbb{N}^{n}$ with $a_{1}+\cdots+a_{n}=m$ one has

$$
\sum_{\operatorname{sig}(u)=\alpha} \operatorname{Tr}\left(\rho_{1}(u)\right)=\sum_{\operatorname{sig}(u)=\alpha} \operatorname{Tr}\left(\rho_{2}(u)\right)
$$

To complete the proof it is enough to show by induction on the partial ordering of words that for each word $w$ in $G$ we have

$$
\operatorname{Tr}\left(\rho_{1}(w)\right)=\operatorname{Tr}\left(\rho_{2}(w)\right) .
$$

When $w=1$ this statement is trivial since both sides equal $N$. Suppose $w \neq 1$ and we have proved our statement for all words $<w$. Let $m=|w|$ and $\operatorname{sig}(w)=$ $\alpha=\left(a_{1}, a_{2}, \ldots\right)$. For each word $u$ with $\operatorname{sig}(u)=\alpha$ let $\tilde{u}$ be a word in echelon form 
such that $u \leadsto \tilde{u}$, as per the Ordering Theorem. Thus, by Lemma 6.3 we have $\operatorname{ct}(w)=\operatorname{ct}(u) \geq_{\text {lex }} \operatorname{ct}(\tilde{u})$. Let

$$
M=\mid\{u \mid \operatorname{sig}(u)=\alpha \text { and } \operatorname{ct}(\tilde{u})=\operatorname{ct}(w)\} \mid .
$$

Since $u$ and $\tilde{u}$ belong to the same conjugacy class in $G$, we can rewrite equation (8.3) as follows:

$$
\begin{aligned}
\sum_{\substack{\operatorname{sig}(u)=\alpha \\
\tilde{u}<w}} \operatorname{Tr}\left(\rho_{1}(\tilde{u})\right) & +\sum_{\substack{\operatorname{sig}(u)=\alpha \\
\operatorname{ct}(\tilde{u})=\operatorname{ct}(w)}} \operatorname{Tr}\left(\rho_{1}(\tilde{u})\right) \\
& =\sum_{\substack{\operatorname{sig}(u)=\alpha \\
\tilde{u}<w}} \operatorname{Tr}\left(\rho_{2}(\tilde{u})\right)
\end{aligned}
$$

If $\tilde{w}<w$ then by induction hypothesis we get

$$
\operatorname{Tr}\left(\rho_{1}(w)\right)=\operatorname{Tr}\left(\rho_{1}(\tilde{w})\right)=\operatorname{Tr}\left(\rho_{2}(\tilde{w})\right)=\operatorname{Tr}\left(\rho_{2}(w)\right),
$$

so we may assume that $\operatorname{ct}(w)=\operatorname{ct}(\tilde{w})$. Also, note that, in type $D_{n+1}$, if $a_{n}=0$ (resp. $\left.a_{n+1}=0\right)$ and $\operatorname{sig}(u)=\alpha$, then by the nature of the possible admissible transformations, $\tilde{u}$ does not involve $g_{n}$ (resp. $g_{n+1}$ ). Therefore, in view of Remark 7.3 and Proposition 7.4 in all possible cases for $u$ with $\operatorname{sig}(u)=\alpha$ and $\operatorname{ct}(\tilde{u})=\operatorname{ct}(w)$ we must have $\tilde{u}$ in the same conjugacy class as $w$. Therefore $\operatorname{Tr}\left(\rho_{i}(\tilde{u})\right)=\operatorname{Tr}\left(\rho_{i}(w)\right)$ hence

$$
\sum_{\substack{\operatorname{sig}(u)=\alpha \\ \operatorname{ct}(\tilde{u})=\operatorname{ct}(w)}} \operatorname{Tr}\left(\rho_{i}(\tilde{u})\right)=M \operatorname{Tr}\left(\rho_{i}(w)\right)
$$

for $i=1,2$. Since by the induction hypothesis $\operatorname{Tr}\left(\rho_{1}(\tilde{u})\right)=\operatorname{Tr}\left(\rho_{2}(\tilde{u})\right)$ whenever $\tilde{u}<w$, equation (8.4) reduces to

$$
M \operatorname{Tr}\left(\rho_{1}(w)\right)=M \operatorname{Tr}\left(\rho_{2}(w)\right)
$$

and the desired conclusion is immediate.

We also note the following result which was obtained during the course of establishing equality (8.3) in the proof above.

Theorem 8.5. Let $G$ be any finitely generated group with generators $g_{1}, \ldots, g_{n}$, and let $\rho_{1}$ and $\rho_{2}$ be two finite dimensional representations of $G$. If the equality (8.1) holds, then for every $\left(k_{1}, \ldots, k_{n}\right) \in \mathbb{N}^{n}$ we have

$$
\sum_{\operatorname{sig}(w)=\left(k_{1}, \ldots, k_{n}\right)} \chi_{\rho_{1}}(w)=\sum_{\operatorname{sig}(w)=\left(k_{1}, \ldots, k_{n}\right)} \chi_{\rho_{2}}(w),
$$

where $w$ denotes a word in the alphabet given by the set $\left\{g_{1}, \ldots, g_{n}\right\}$.

\section{EXAmple: $\widetilde{C}_{2}$}

Recall that $\widetilde{C}_{2}$ is the Coxeter group with three generators $b_{1}, b_{2}, b_{3}$ that satisfy the relations:

$$
b_{j}^{2}=1, j=1,2,3 ; \quad\left(b_{1} b_{2}\right)^{4}=\left(b_{2} b_{3}\right)^{4}=1 ; \quad b_{1} b_{3}=b_{3} b_{1} .
$$


It is well known that this group is affine, that is, it contains an abelian normal subgroup such that the quotient group is finite. In this particular case it is easy to see that this normal abelian subgroup is the following: let

$$
r_{1}=b_{1} b_{2} b_{3} b_{2}, \quad r_{2}=b_{2} b_{1} b_{2} b_{3} \text {. }
$$

Let us check that the group generated by $r_{1}$ and $r_{2}$ is an abelian normal subgroup of $\widetilde{C}_{2}$. First we observe that the relations (9.1) imply

$$
\begin{aligned}
b_{1} r_{1} & =r_{1}^{-1} b_{1} \\
b_{2} r_{1} & =r_{2} b_{2}, \\
b_{3} r_{1} & =r_{1} b_{3}, \\
b_{1} r_{2} & =r_{2} b_{1} \\
b_{2} r_{2} & =r_{1} b_{2}, \\
b_{3} r_{2} & =r_{2}^{-1} b_{3} \\
b_{3} r_{2}^{-1} & =r_{2} b_{3}
\end{aligned}
$$

We now can use these last relations to establish that $r_{1}$ and $r_{2}$ commute:

$r_{1} r_{2}=r_{1} b_{2} b_{1} b_{2} b_{3}=b_{2} r_{2} b_{1} b_{2} b_{3}=b_{2} b_{1} r_{2} b_{2} b_{3}=b_{2} b_{1} b_{2} r_{1} b_{3}=b_{2} b_{1} b_{2} b_{3} r_{1}=r_{2} r_{1}$.

Now, relations (9.2) imply that

$$
\begin{aligned}
& b_{1} r_{1} b_{1}=r_{1}^{-1} \\
& b_{2} r_{1} b_{2}=r_{2} \\
& b_{3} r_{1} b_{3}=r_{1} \\
& b_{1} r_{2} b_{1}=r_{2} \\
& b_{2} r_{2} b_{2}=r_{1} \\
& b_{3} r_{2} b_{3}=r_{2}^{-1}
\end{aligned}
$$

These last relations show that the subgroup $\mathcal{N}$ of $\widetilde{C}_{2}$ generated by $r_{1}$ and $r_{2}$ is a normal subgroup. We further remark that $b_{1}=b_{2} b_{3} b_{2} r_{1}^{-1}$, and, therefore, the cosets of $b_{2}$ and $b_{3}$ generate $\widetilde{C}_{2} / \mathcal{N}$. We will now show:

Theorem 9.3. If for two finite dimensional unitary representations $\rho_{1}$ and $\rho_{2}$ of the affine Coxeter group $\widetilde{C}_{2}$ we have

$$
\begin{aligned}
& \sigma_{p}\left(\rho_{1}\left(b_{2}\right), \rho_{1}\left(b_{3}\right), \rho_{1}\left(r_{1}\right), \rho_{1}\left(r_{2}\right), \rho_{1}\left(r_{1}^{-1}\right), \rho_{1}\left(r_{2}^{-1}\right)\right) \\
& =\sigma_{p}\left(\rho_{2}\left(b_{2}\right), \rho_{2}\left(b_{3}\right), \rho_{2}\left(r_{1}\right), \rho_{2}\left(r_{2}\right), \rho_{2}\left(r_{1}^{-1}\right), \rho_{2}\left(r_{2}^{-1}\right)\right),
\end{aligned}
$$

then for these representations $\chi_{\rho_{1}}=\chi_{\rho_{2}}$.

Proof. First we note that the subgroup generated by $b_{2}$ and $b_{3}$ is the dihedral group $I(4)=B_{2}$. The only words in echelon form here are $1, b_{2}, b_{3}, b_{2} b_{3}, b_{2} b_{3} b_{2}$, and $b_{2} b_{3} b_{2} b_{3}$. Note that $b_{3}$ and $b_{2} b_{3} b_{2}$ belong to the same conjugacy class. Since every element of $G$ can be written as $w r_{1}^{m_{1}} r_{2}^{m_{2}}$ for some word $w$ in $b_{2}$ and $b_{3}$, formulae (9.2) and Theorem 7.5 imply that every element in $\widetilde{C}_{2}$ has in its conjugacy class a word in the form

$$
b_{2}^{k_{1}} b_{3}^{l_{1}} b_{2}^{k_{2}} b_{3}^{k_{2}} r_{1}^{m_{1}} r_{2}^{m_{2}}
$$


where $k_{1}, k_{2}, l_{1} \in\{0,1\}, k_{1} \geq k_{2}, l_{1} \geq k_{2}$, and $m_{1}, m_{2} \in \mathbb{Z}$. In other words, each word in $\widetilde{C}_{2}$ has in its conjugacy class one of the words

$$
\begin{aligned}
& w_{0}\left(m_{1}, m_{2}\right)=r_{1}^{m_{1}} r_{2}^{m_{2}}, \\
& w_{1}\left(m_{1}, m_{2}\right)=b_{2} r_{1}^{m_{1}} r_{2}^{m_{2}}, \\
& w_{2}\left(m_{1}, m_{2}\right)=b_{3} r_{1}^{m_{1}} r_{2}^{m_{2}}, \\
& w_{3}\left(m_{1}, m_{2}\right)=b_{2} b_{3} r_{1}^{m_{1}} r_{2}^{m_{2}}, \quad \text { or } \\
& w_{4}\left(m_{1}, m_{2}\right)=b_{2} b_{3} b_{2} b_{3} r_{1}^{m_{1}} r_{2}^{m_{2}} .
\end{aligned}
$$

Thus, we are to show that if

$$
\begin{aligned}
& \sigma\left(-I, \rho_{1}\left(b_{2}\right), \rho_{1}\left(b_{3}\right), \rho_{1}\left(r_{1}\right), \rho_{1}\left(r_{2}\right), \rho_{1}\left(r_{1}^{-1}\right), \rho_{1}\left(r_{2}^{-1}\right)\right) \\
& =\sigma\left(-I, \rho_{2}\left(b_{2}\right), \rho_{2}\left(b_{3}\right), \rho_{2}\left(r_{1}\right), \rho_{2}\left(r_{2}\right), \rho_{2}\left(r_{1}^{-1}\right), \rho_{2}\left(r_{2}^{-1}\right)\right),
\end{aligned}
$$

then for every $m_{1}, m_{2} \in \mathbb{Z}$ and every $j=0,1,2,3,4$

$$
\chi_{\rho_{1}}\left(w_{j}\left(m_{1}, m_{2}\right)\right)=\chi_{\rho_{2}}\left(w_{j}\left(m_{1}, m_{2}\right)\right) .
$$

A similar argument to the one in Theorem 8.5 shows that for every $\eta \in \mathbb{N}^{6}$ with $\eta=\left(n_{1}, n_{2}, n_{3}, n_{4}, n_{5}, n_{6}\right)$

$$
\sum_{\operatorname{sig}(w)=\eta} \chi_{\rho_{1}}(w)=\sum_{\operatorname{sig}(w)=\eta} \chi_{\rho_{2}}(w) .
$$

where the sums are taken over all words comprised of $n_{1} \quad b_{2}$-s, $n_{2} \quad b_{3}$-s, $n_{3} \quad r_{1}$-s, $n_{4} \quad r_{2}$-s, $n_{5} \quad r_{1}^{-1}$-s, and $n_{6} \quad r_{2}^{-1}$-s.

In particular, using circular transformation if necessary, we see that in the cases $n_{1}=1, n_{2}=n_{5}=n_{6}=0$ all terms in each of the sums (9.5) are the same. Therefore, for all $m_{1}, m_{2} \geq 0$

$$
\chi_{\rho_{1}}\left(w_{1}\left(m_{1}, m_{2}\right)\right)=\chi_{\rho_{2}}\left(w_{1}\left(m_{1}, m_{2}\right)\right) .
$$

A similar argument shows that the same equality holds for $m_{1} \geq 0, m_{2} \leq 0, m_{1} \leq$ $0, m_{2} \geq 0, m_{1}, m_{2} \leq 0$, and, hence, (9.6) holds for all $m_{1}, m_{2} \in \mathbb{Z}$.

Applying the same reasoning to the case $n_{1}=0, n_{2}=1$ we obtain

$$
\chi_{\rho_{1}}\left(w_{j}\left(m_{1}, m_{2}\right)\right)=\chi_{\rho_{2}}\left(w_{j}\left(m_{1}, m_{2}\right)\right) .
$$

holds for $j=2$ as well.

Let us show that (9.7) holds for $j=3$. We will prove it by induction in $s=$ $\left|m_{1}\right|+\left|m_{2}\right|$. If $s=0, w_{3}(0,0)$ belongs to the subgroup generated by $b_{2}$ and $b_{3}$. Since the joint spectrum of $\rho_{j}\left(b_{2}\right), \rho_{j}\left(b_{3}\right)$ and the identity is the intersection of $\sigma\left(-I, \rho_{1}\left(b_{2}\right), \rho_{1}\left(b_{3}\right), \rho_{1}\left(r_{1}\right), \rho_{1}\left(r_{2}\right), \rho_{1}\left(r_{1}^{-1}\right), \rho_{1}\left(r_{2}^{-1}\right)\right)$ with the plane $\left\{x_{3}=x_{4}=\right.$ $\left.x_{5}=x_{6}=0\right\}$, we see that

$$
\sigma\left(-I, \rho_{1}\left(b_{2}\right), \rho_{1}\left(b_{3}\right)\right)=\sigma\left(-I, \rho_{2}\left(b_{2}\right), \rho_{2}\left(b_{3}\right)\right),
$$

so by our previous results

$$
\chi_{\rho_{1}}\left(b_{2} b_{3}\right)=\chi_{\rho_{2}}\left(b_{2} b_{3}\right) .
$$

Now, let us fix $m_{1}, m_{2} \geq 0$ with $m_{1}+m_{2}>0$, and consider $n_{1}=n_{2}=1, n_{3}=$ $m_{1}, n_{4}=m_{2}, n_{5}=n_{6}=0$. In each side of (9.5) there are characters of words in one of two forms:

$$
r_{1}^{q_{1}} r_{2}^{t_{1}} b_{2} r_{1}^{q_{2}} r_{2}^{t_{2}} b_{3} r_{1}^{q_{3}} r_{2}^{t_{3}}, \quad \text { or } \quad r_{1}^{q_{1}} r_{2}^{t_{1}} b_{3} r_{1}^{q_{2}} r_{2}^{t_{2}} b_{2} r_{1}^{q_{3}} r_{2}^{t_{3}},
$$


where $q_{j}, t_{j} \geq 0, q_{1}+q_{2}+q_{3}=m_{1}, t_{1}+t_{2}+t_{3}=m_{2}$ (we observe that the order of $r_{1}$ and $r_{2}$ does not matter since they commute).

Using circular transformations shows that each word that appears in the second form in (9.8) is in the same conjugacy class as the first one, of course with different $q_{j}$ and $t_{j}$ which still sum up to $m_{1}$ and $m_{2}$ respectively. Thus, it suffices to show the equality of characters for words in the first form in (9.8).

We use circular transformation to get

$$
\begin{aligned}
r_{1}^{q_{1}} r_{2}^{t_{1}} b_{2} r_{1}^{q_{2}} r_{2}^{t_{2}} b_{3} r_{1}^{q_{3}} r_{2}^{t_{3}} & \sim b_{2} r_{1}^{q-2} r_{2}^{t_{2}} b_{3} r_{1}^{q_{3}} r_{2}^{t_{3}} r_{1}^{q_{1}} r_{2}^{t_{1}} \\
& =b_{2} r_{1}^{q_{2}} r_{2}^{t_{2}} b_{3} r_{1}^{q_{1}+q_{3}} r_{2}^{t_{1}+t_{3}} .
\end{aligned}
$$

By (9.2) $r_{2} b_{3}=b_{3} r_{2}^{-1}$, so we can get from (9.9)

$$
r_{1}^{q_{1}} r_{2}^{t_{1}} b_{2} r_{1}^{q_{2}} r_{2}^{t_{2}} b_{3} r_{1}^{q_{3}} r_{2}^{t_{3}} \sim b_{2} r_{1}^{q_{2}} b_{3} r_{1}^{q_{1}+q_{3}} r_{2}^{t_{1}-t_{2}+t_{3}}
$$

Since by (9.2) $r_{1}$ and $b_{3}$ commute, we finally obtain

$$
r_{1}^{q_{1}} r_{2}^{t_{1}} b_{2} r_{1}^{q_{2}} r_{2}^{t_{2}} b_{3} r_{1}^{q_{3}} r_{2}^{t_{3}} \sim b_{2} b_{3} r_{1}^{q_{1}+q_{2}+q_{3}} r_{2}^{t_{1}-t_{2}+t_{3}}=b_{2} b_{3} r_{1}^{m_{1}} r_{2}^{t_{1}-t_{2}+t_{3}} .
$$

If $\left|t_{1}-t_{2}+t_{3}\right|<m_{2}$, by induction

$$
\chi_{\rho_{1}}\left(r_{1}^{q_{1}} r_{2}^{t_{1}} b_{2} r_{1}^{q_{2}} r_{2}^{t_{2}} b_{3} r_{1}^{q_{3}} r_{2}^{t_{3}}\right)=\chi_{\rho_{2}}\left(r_{1}^{q_{1}} r_{2}^{t_{1}} b_{2} r_{1}^{q_{2}} r_{2}^{t_{2}} b_{3} r_{1}^{q_{3}} r_{2}^{t_{3}}\right) .
$$

Of course, for non-negative $t_{1}, t_{2}, t_{3}$

$$
t_{1}+t_{2}+t_{3}=\left|t_{1}-t_{2}+t_{3}\right| \Longrightarrow t_{2}=0 \text {, or } t_{1}=t_{3}=0 .
$$

We will now show that every term in (9.5) that are not conjugates of words in the form (9.8) with a smaller $m_{1}+m_{2}$ is in the same conjugacy class as $b_{2} b_{3} r_{1}^{m_{1}+m_{2}}$. Of course, this together with (9.5) and (9.11) will imply that (9.4) holds for $j=3$.

By (9.12) for such a word either $t-2=0$, that is the word is in the form

$$
\begin{aligned}
r_{1}^{q_{1}} r_{2}^{t} b_{2} r_{1}^{q_{2}} b_{3} r_{1}^{q_{3}} r_{2}^{m_{2}-t} & =r_{1}^{q_{1}} r_{2}^{t} b_{2} b_{3} r_{1}^{m_{1}-q_{1}} r_{2}^{m_{2}-t} \\
& \sim b_{2} b_{3} r_{1}^{m_{1}} r_{2}^{m_{2}},
\end{aligned}
$$

(here we used the fact that $b_{3}$ and $r_{1}$ commute and a circular transformation), or $t_{1}=t_{3}=0$, so the word is

$$
\begin{aligned}
r_{1}^{q_{1}} b_{2} r_{1}^{q_{2}} r_{2}^{m_{2}} b_{3} r_{1}^{q_{3}} & =r_{1}^{q_{1}} b_{2} r_{2}^{m_{2}} r_{1}^{q_{1}} b_{3} r_{1}^{q_{3}} \\
& =r_{1}^{q_{1}} b_{2} r_{2}^{m_{2}} b_{3} r_{1}^{q_{2}+q_{3}} \\
& \sim b_{2} r_{2}^{m_{2}} b_{3} r_{1}^{m_{1}},
\end{aligned}
$$

here we again we used the commuting of $b_{3}$ and $r_{1}$ and a circular transformation that moved $r_{1}^{q_{1}}$ to the end of the word.

Now, by (9.2) $b_{2} r_{2}=r_{1} b_{2}$ and $r_{2} b_{2}=b_{2} r_{1}$, therefore, in (9.13) we have

$$
b_{2} b_{3} r_{1}^{m_{1}} r 2^{m_{2}} \sim r_{2}^{m_{2}} b_{2} b_{3} r_{1}^{m_{1}}=b_{2} r_{1}^{m_{2}} b_{3} r_{1}^{m_{1}}=b_{2} b_{3} r_{1}^{m_{1}+m_{2}} \text {. }
$$

For (9.14) we have

$$
b_{2} r_{2}^{m_{2}} b_{3} r_{1}^{m_{1}}=r_{1}^{m_{2}} b_{2} b_{3} r_{1}^{m_{1}} \sim b_{2} b_{3} r_{1}^{m_{1}+m_{2}} .
$$

This finishes the proof of (9.4) for $j=3$ and $m_{1}, m_{2} \geq 0$. The proofs in the cases when one of, or both $m_{1}$ and $m_{2}$ are negative are practically identical with the only difference being that for $m_{1}<0 \quad r_{1}$ is replaced with $r_{1}^{-1}$, and $r_{2}$ is replaced with $r_{2}^{-1}$ when $m_{2}<0$. 
We now prove that (9.4) holds for $j=4$. Again, for every pair of representations with the same joint spectrum of $b_{2}, b_{3}, r_{1}, r_{2}, r_{1}^{-1}, r_{2}^{-1}$ the restrictions of these representations to the subgroup generated by $b_{2}$ and $b_{3}$ are unitary equivalent so

$$
\chi_{\rho_{1}}\left(b_{2} b_{3} b_{2} b_{3}\right)=\chi_{\rho_{2}}\left(b_{2} b_{3} b_{2} b_{3}\right) .
$$

Next we observe that relations (9.2) imply

$$
b_{2} r_{1} b_{2}=r_{2}, b_{2} r_{2} b_{2}=r_{1}, b_{2} r_{1}^{-1} b_{2}=r_{2}^{-1}, b_{2} r_{2}^{-1} b_{2}=r_{1}^{-1},
$$

and, therefore,

$$
b_{2} r_{1}^{k} b_{2}=r_{2}^{k}, b_{2} r_{2}^{k} b_{2}=r_{1}^{k}, k \in \mathbb{Z}
$$

Relations (9.15) imply

$$
b_{2} b_{3} b_{2} b_{3} r_{1}^{k} r_{2}^{l} \sim b_{2} b_{3} b_{2} b_{3} r_{1}^{l} r_{2}^{k}, k, l \in \mathbb{Z} .
$$

Indeed,

$$
\begin{aligned}
b_{2} b_{3} b_{2} b_{3} r_{1}^{k} r_{2}^{l}=b_{2} b_{3} b_{2} b_{3} b_{2} r_{2}^{k} b_{2} b_{2} r_{1}^{l} b_{2}=b_{2} b_{3} b_{2} b_{3} b_{2} r_{2}^{k} r_{1}^{l} b_{2} \\
=b_{3} b_{2} b_{3} r_{2}^{k} r_{1}^{l} b_{2} \sim b_{2} b_{3} b_{2} b_{3} r_{1}^{l} r_{2}^{k} .
\end{aligned}
$$

We also remark that

$$
b_{2} b_{3} b_{2} b_{3} r_{1}^{k} r_{2}^{l} \sim b_{2} b_{3} b_{2} b_{3} r_{1}^{k-2} r_{2}^{l}
$$

and, similarly

$$
b_{2} b_{3} b_{2} b_{3} r_{1}^{k} r_{2}^{l} \sim b_{2} b_{3} b_{2} b_{3} r_{1}^{k} r_{2}^{l-2}
$$

Verification of (9.17) goes as follows:

$$
\begin{aligned}
b_{2} b_{3} b_{2} b_{3} r_{1}^{k} r_{2}^{l} & =b_{2} b_{3} b_{2} b_{3} r_{1}^{k-1} r_{2}^{l} r_{1} \\
& \sim r_{1} b_{2} b_{3} b_{2} b_{3} r_{1}^{k-1} r_{2}^{l} \\
& =b_{2} r_{2} b_{3} b_{2} b_{3} r_{1}^{k-1} r_{2}^{l} \\
& =b_{2} b_{3} r_{2}^{-1} b_{2} b_{3} r_{1}^{k-1} r_{2}^{l} \\
& =b_{2} b_{3} b_{2} r_{1}^{-1} b_{3} r_{1}^{k-1} r_{2}^{l} \\
& =b_{2} b_{3} b_{2} b_{3} r_{1}^{k-2} r_{2}^{l} .
\end{aligned}
$$

Relation (9.18) is an immediate consequence of (9.17) and (9.16). Now, (9.17) and (9.18) show that if $|k|+|l| \geq 2$, and $(k, l) \neq( \pm 1, \pm 1)$, then

$$
b_{2} b_{3} b_{2} b_{3} r_{1}^{k} r_{2}^{l} \sim b_{2} b_{3} b_{2} b_{3} r_{1}^{k_{1}} r_{2}^{l_{1}} \quad \text { with } \quad\left|k_{1}\right|+\left|l_{1}\right|<|k|+|l| .
$$

Suppose that $m_{1}, m_{2} \geq 0$. Each term in (9.5) with $n_{1}=2, n_{2}=2, n_{3}=m_{1}, n_{4}=$ $m_{2}, n_{5}=n_{6}=0$ is in the form

$$
r_{1}^{q_{1}} r_{2}^{t_{1}} b_{j_{1}} r_{1}^{q_{2}} r_{2}^{t_{2}} b_{j_{2}} r_{1}^{q_{3}} r_{2}^{t 3} b_{j_{3}} r_{1}^{q_{4}} r_{2}^{t_{4}} b_{j_{4}} r_{1}^{q_{5}} r_{2}^{t_{5}},
$$

where $q_{k}, t_{k} \geq 0, \sum q_{k}=m_{1}, \sum t_{k}=m_{2}, j_{k}=\{2,3\}$ and there are two $b_{2}$ among $b_{j_{k}}$ and two $b_{3}$. Observe that, if for some $k=1,2,3 \quad j_{k}=j_{k+1}$, then formulae (9.2) show that the word is a conjugate of either $w_{3}\left(l_{1}, l_{2}\right)$, or of $w_{0}\left(l_{1}, l_{2}\right)$ for some $l_{1}, l_{2}$. Since for such words we have already proven the equality of the characters, the sums of terms in (9.5) with $j_{k} \neq j_{k+1}$ on the left and on the right are the same. Each of such therms is in the form (9.20) with $b_{j_{1}}=b_{2}, b_{j_{2}}=b_{3}, b_{j_{3}}=b_{2}, b_{j_{4}}=b_{3}$, or with $b_{j_{1}}=b_{3}, b_{j_{2}}=b_{2}, b_{j_{3}}=b_{3}, b_{j_{4}}=b_{2}$. Using circular transformations we easily see that the latter one is a conjugate of the former (possibly with different 
$q$-s and $t$-s). As it was mentioned above, relations (9.2) shows that each such term is a conjugate of

for some $k$ and $l$.

$$
b_{2} b_{3} b_{2} b_{3} r_{1}^{k} r_{2}^{l}
$$

A similar argument shows that the same conjugacy relation holds for arbitrary $m_{1}, m_{2} \in \mathbb{Z}$.

Now we use an induction argument in $\left|m_{1}\right|+\left|m_{2}\right|$. We have already proven that (9.4) holds for $w_{4}(0,0)$. Relation (9.19) shows that it suffices to prove it for $w_{4}( \pm 1,0), w_{4}(0, \pm 1)$ and $w_{4}( \pm 1, \pm 1)$.

Obviously, (9.16)-9.18) imply

$$
\begin{array}{r}
w_{4}(1,0) \sim w_{4}(0,1) \sim w_{4}(-1,0) \sim w_{4}(0,-1), \\
w_{4}(1,1) \sim w_{4}(-1,1) \sim w_{4}(1,-1) \sim w_{4}(-1,-1) .
\end{array}
$$

All terms in relation (9.5) with $n_{1}=2, n_{2}=2, n_{3}=1, n_{4}=n_{5}=n_{5}=0$ that are not conjugates of $w_{3}(k, l)$ or $w_{0}(k, l)$ are

$$
\begin{array}{ll}
r_{1} b_{2} b_{3} b_{2} b_{3}, & r_{1} b_{3} b_{2} b_{3} b_{2}, \\
b_{2} r_{1} b_{3} b_{2} b_{3}, & b_{3} r_{1} b_{2} b_{3} b_{2}, \\
b_{2} b_{3} r_{1} b_{2} b_{3}, & b_{3} b_{2} r_{1} b_{3} b_{2}, \\
b_{2} b_{3} b_{2} r_{1} b_{3}, & b_{3} b_{2} b_{3} r_{1} b_{2}, \\
b_{2} b_{3} b_{2} b_{3} r_{1}, & b_{3} b_{2} b_{3} b_{2} r_{1} .
\end{array}
$$

It is easy to see using (9.2) that each of them is a conjugate of one of $w_{4}( \pm 1,0)$ or $w_{4}(0, \pm 1)$. By (9.21) all of them are in the same conjugate class and, therefore, (9.4) implies

$$
\chi_{\rho_{1}}\left(w_{4}( \pm 1,0)\right)=\chi_{\rho_{2}}\left(w_{4}( \pm 1,0)\right), \chi_{\rho_{1}}\left(w_{4}(0, \pm 1)\right)=\chi_{\rho_{2}}\left(w_{4}(0, \pm 1)\right) .
$$

Similarly, we can show that each term in (9.5) corresponding to $n_{1}=2, n_{2}=2, n_{3}=$ $n_{4}=1, n_{5}=n_{6}=0$ is either a conjugate of $w_{4}(0,0)$, or of $w_{4}( \pm 2,0) \sim w_{4}(0,0)$, or of $w_{4}\left(0, \pm 20 \sim w_{4}(0,0)\right.$, or $w_{4}( \pm 1, \pm 1)$. For the first three the equality of characters has been established. For the rest this equality follows from (9.4) and (9.22).

\section{REFERENCES}

[1] A. Bjorner and F. Brenti, Combinatorics of Coxeter Groups, Springer, 2005.

[2] J. P. Bannon, P. Cade, and R. Yang, On the spectrum of Banach algebra-valued entire functions, Ill. J. Math., 55 (4), (2011), 1455-1465.

[3] P. Cade and R. Yang, Projective spectrum and cyclic cohomology, J. Funct. Anal., 265 (9) (2013), 1916-1933.

[4] F. Catanese, Babbage's conjecture, contact surfaces, symmentric determinantal varieties and applications, Invent. Math., 63 (1981), 433-465.

[5] I. Chagouel, M. Stessin, and K. Zhu, Geometric spectral theory for compact operators, Trans. Amer. Math. Soc., 368 (3) (2016), 1559-1582.

[6] H. M. S. Coxeter, Discrete groups generated by reflections, Ann. of Math. (2), 35 (3) (1934), $588-621$.

[7] H. M. S. Coxeter, The complete enumeration of finite groups of the form $r_{i}^{2}=\left(r_{i} r_{j}\right)^{k_{i j}}=1$, J. London Mat. Soc., 10 (1) (1935), 21-25.

[8] L. E. Dickson, An elementary exposition of Frobenius' theory of group characters and group determinants, Ann. of Math. (2), 4 (1902), 25-49; also in Mathematical papers, Vol. II, Chelsea, New York, 1975, 737-761.

[9] L. E. Dickson, On the group defined for any given field by the multiplication table of any given finite group, Trans. Amer. Math. Soc., 3 (1902), 377-382; also in Mathematical Papers, Vol. II, Chelsea, New York, 1975, 75-91. 
[10] L. E. Dickson, Modular theory of group-matrices, Trans. Amer. Math. Soc., 8 (1907), 389398; also in Mathematical Papers, Vol. II, Chelsea, New York, 1975, 251-260.

[11] L. E. Dickson, Modular theory of group-characters, Bull. Amer. Math. Soc., 13 (1907), 477488; also in Mathmatical Papers, Vol. IV, Chelsea, New York, 1975, 535-546.

[12] L. E. Dickson, Determination of all general homogeneous polynomials expressible as determinants with linear elements, Trans. Amer. Math. Soc., 22 (1921), 167-179.

[13] I. Dolgachev, Classical Algebraic Geometry: A modern View, Cambridge University Press, 2012.

[14] R. G. Douglas and R. Yang, Hermitian geometry on reslovent set (I), Proceedings of the International Workshop on Operator Theory (IWOTA 2016), to appear.

[15] R. G. Douglas and R. Yang, Hermitian geometry on resolvent set (II), preprint.

[16] E. Formanek and D. Sibley, The group determinant determines the group, Proc. Amer. Math. Soc., 112 (1991), 649-656.

[17] F. G. Frobenius, Über vertauschbare Matrizen, Sitz. Kön. Preuss. Akad. Wiss. Berlin (1896) 601-614; also in Gesammelte Abhandlungen, Band II, Springer-Verlag, New York, 1968, 705718.

[18] F. G. Frobenius, Über Gruppencharaktere, Sitz. Kön. Preuss. Akad. Wiss. Berlin (1896) 985-1021; also in Gesammelte Abhandlungen, Band III, Springer-Verlag, New York, 1968, $1-37$.

[19] F. G. Frobenius, Über die Primfactoren der Gruppendeterminante. Sitz. Kön. Preuss. Akad. Wiss. Berlin (1896) 1343-1382; also in Gesammelte Abhandlungen, Band III, SpringerVerlag, New York, 1968, 38-77.

[20] M. Geck and G. Pfeiffer, Characters of Finite Coxeter Groups and Iwahory-Hecke Algebras, Calderon Press, Oxford, 2000.

[21] P. Gonzalez-Vera and M.I. Stessin, Joint spectra of Toeplitz operators and optimal recovery of analytic functions, Constructive Approximation, 36 (1) (2012), 53-82.

[22] R. Grigorchuk and R.Yang, Joint spectrum and infinite dihedral group, Proc. Steklov Inst. Math., 297 (2017), 145-178.

[23] J. W. Helton, S. A. McCullogh, and V. Vinnikov, Non-commutative convexity arises from linear matrix inequalities, J. Funct. Anal., 240 (1) (2006), 105-191.

[24] J. W. Helton and V. Vinnikov, Linear matrix inequality representation set, Comm. Pure and Appl. Math., 60, (2007), 654-674.

[25] W. He, X. Wang, and R. Yang, Projective spectrum and kernel bundle (II), J. Oper. Theory, 297 (2017), 417-433.

[26] H.-J. Hoehnke and K. W. Johnson, The 1-, 2-, and 3-characters determine a group, Bull. Amer. Math. Soc., 27 (1992), 243-245.

[27] H.-J. Hoehnke and K. W. Johnson, $k$-Characters and group invariants, Comm. in Algebra, 26 (1998), 1-27.

[28] J. E. Humphreys, Reflection Groups and Coxeter Groups, Cambridge Studies in Advanced Mathematics 10, Cambridge University Press, 1992.

[29] K. W. Johnson, On the group determinant, Math. Proc. Cambridge Philos. Soc., 109 (1991), 299-311.

[30] D. Kerner and V. Vinnikov, Determinantal representations of singular hypersurfaces in $\mathbb{P}^{n}$, Adv. Math., 231 (2012), 1619-1654

[31] P. D. Lax, Functional Analysis, Wiley, 2002.

[32] T. S. Motzkin and O. Taussky, Pairs of matrices with property L, Trans. Amar. Math. Soc., 73 (1952), 108-114.

[33] T. Rivlin, The Chebyshev polynomials, Pure and Applied Mathematics, Wiley-Interscience, New York-London-Sydney, 1974.

[34] T. G. Room, Self-transformations of determinantal quadric surfaces I, Proc. London Mat. Soc., 51 (2) (1950), 348-361.

[35] J. P. Serre, Linear Representations of Finite Groups, Graduate Texts in Mathematics 42, Springer-Verlag, New York-Heidelberg, 1977.

[36] M. Stessin, R. Yang, K. Zhu, Analyticity of a joint spectrum and multivariable analytic Fredholm theorem, New York J. Math., 17a (2011), 39-44.

[37] M. I. Stessin and A. B. Tchetnev, Spectral algebraic curves and decomposable operator tuples, arxiv: 1509.06274

[38] M. I. Stessin and A. B. Tchernev, in preparation. 
[39] V. Vinnikov, Complete description of determinantal representations of smooth irreducible curves, Lin. Albebra and Appl., 125 (1989), 103-140.

[40] R. Yang, Projective spectrum in Banach algebras, J. Topol. Anal., 1 (2009), 289-306.

Department of Mathematics and Statistics, University of Toledo, Toledo, OH 43606, USA

E-mail address: zcuckovi@math.utoledo.edu

Department of Mathematics and Statistics, University at Albany, SUny, Albany, NY 12222, USA

E-mail address: mstessin@albany.edu

Department of Mathematics and Statistics, University at Albany, SUny, Albany, NY 12222, USA

E-mail address: atchernev@albany.edu 